\title{
Gas accretion on spiral galaxies: Bar formation and renewal
}

\author{
F. Bournaud ${ }^{1}$ and F. Combes ${ }^{2}$ \\ ${ }^{1}$ École Normale Supérieure, 45 rue d'Ulm, 75005 Paris, France \\ 2 Observatoire de Paris, DEMIRM, 61 Av. de l'Observatoire, 75014 Paris, France \\ Received 14 January 2002 / Accepted 17 June 2002

\begin{abstract}
The effects of gas accretion on spiral disk dynamics and stability are studied through $N$-body simulations, including star formation and gas/stars mass exchange. The detailed processes of bar formation, bar destruction and bar re-formation are followed, while in the same time the disk to bulge ratio is varying. The accreted gas might be first prevented to flow inwards to the center by the bar gravity torques, which maintains it to the outer Lindblad resonance. While the first bar is weakening, the accreted gas replenishes the disk, increasing the disk-to-bulge ratio, and the disk self-gravity. A second bar is then unstable, with a higher pattern speed, due both to the increased mass, and shorter bar length. Three or four bar episodes have been followed over a Hubble time. Their strength is decreasing with time, while their pattern speed is increasing. Detailed balance of the angular momentum transfer and evolution can account for these processes. The gas recycled through star formation, and rejected through stellar mass loss plays also a role in the disk dynamics. Implications on the spiral galaxy dynamics and evolution along the Hubble sequence, and as a function of redshift are discussed.
\end{abstract}

Key words. galaxies: evolution - galaxies: spiral - methods: $N$-body simulations

\section{Introduction}

Bars are an essential feature in galaxy evolution. About twothirds of spiral galaxies are barred (de Vaucouleurs 1963), one third being strongly barred (SB), the other third mildly barred (SAB). In the near-infrared, where the central structure is unveiled from dust, the fraction is even higher (Eskridge et al. 2000).

In the last decade, it has been realized that bars are not long lived features, in particular in gaseous spiral disks, and that galaxies are not frozen for a Hubble time in their morphological class: a bar can be destroyed by large radial gas inflow and mass accumulation in the center (Hasan \& Norman 1990; Pfenniger \& Norman 1990; Hasan et al. 1993; Friedli \& Benz 1993). The mass concentration destroys the orbital structure that supported the bar, and this begins through the creation of two strong inner Lindblad resonances (ILR). The process is initiated by the strong gravity torques exerted by the bar on the gaseous spiral arms, which decrease afterwards as the bar weakens. It can lead to the decoupling of a secondary bar, embedded into the primary, just inside its ILR (Friedli \& Martinet 1993; Combes 1994), and this is likely to weaken the primary bar.

The bar phenomenon can then be a self-regulated process (e.g. Combes 2000). A tantalizing scenario is that a galaxy may have several bar episodes in its life, and that the fraction of barred galaxies in the de Vaucouleurs classification only reflects the time percentage that a spiral galaxy spends as a barred

Send offprint requests to: F. Bournaud, e-mail: bournaud@clipper.ens.fr object. How can a bar be revived after a destruction or weakening event? The disk self-gravity must be enhanced to counteract the stabilizing influence of the bulge and central mass concentration, enforced by the first bar episode. This may be obtained through external gas accretion, that settles in the disk. Significant amounts of matter can be accreted in the life-time of the galaxy (e.g. Katz et al. 1996), and we consider realistic that the mass of a galaxy could double in a few Gyrs. The presence of gaseous warps in practically every spiral galaxy (Sancisi 1983; Briggs 1990) has been interpreted as a sign that a spiral galaxy accretes mass and angular momentum: the amount accreted is such that it changes completely orientation in a typical time-scale of a few Gyrs (Jiang \& Binney 1999).

During its life, a bar changes its pattern speed, and this could be a possible way to trace its age. In dissipationless simulations, the pattern speed decreases in a time-scale of a few Gyrs (Combes \& Sanders 1981). This is also related to the length of the bar. The early bar instability involves central orbits, where the orbital precession rate is higher, then more and more orbits become trapped in the bar, at larger radii, and the bar lengthens and slows down, The transient spiral waves formed in the stellar component transfer the angular momentum outwards. This process is also accelerated by escaping chaotic orbits, around corotation (Pfenniger \& Friedli 1991). If there is a massive spheroidal dark matter halo, concentrated enough to perturb the bar dynamics, the pattern speed of the bar could decrease even further, owing to the dynamical friction (Debattista \& Sellwood 1998). According to the density of dark matter, the time scale could be estimated down to a few $10^{8} \mathrm{yr}$. 
Since bars are observed most of the time to be fast rotators, this puts constraints on the density of dark matter in the central parts of galaxies. Fluctuations of the pattern speed can also be triggered by interacting companions (Gerin et al. 1990; Miwa \& Noguchi 1998), but these remain transient. If gas accretion is able to rejuvenate the bars, it is important to know the implications on the pattern speed, that could be a tracer of the event. This is studied in detail in the presented simulations.

In Sect. 2 we briefly present the code and numerical methods used. In Sects. 3-5 we present the results from our simulations. We give our conclusions in Sect. 6.

\section{Physical model for disk galaxies and numerical techniques}

To explore the influence of gas accretion on the disk dynamics, there are many free parameters to vary; in a first step, to increase computing efficiency, we consider only 2-D models, with high spatial resolution and low CPU cost, with the constraint that the accretion has the same angular momentum direction as the initial galaxy disk. In a second step, with 3-D models, the angular momentum of the accreted matter is varied.

We first describe the two-dimensional models and present the associated results in Sect. 3. We present the threedimensional models, with a lower resolution, in Sect. 4.

\subsection{Disk, bulge and halo}

The galactic disk is first considered as an infinitely flattened two-dimensional component, embedded in a spherical halo, and a bulge partly stabilizes its inner regions. The initial disk is a Toomre disk, with a surface density of the form (Toomre 1963):

$\mu(r)=\mu_{0}\left(1+\frac{r^{2}}{a_{\mathrm{disk}}^{2}}\right)^{-\frac{1}{2}}$.

The scale-length $a_{\text {disk }}$ has been varied between 4 and $6 \mathrm{kpc}$. The disk is initially truncated at a radius $r_{\text {disk }}=15 \mathrm{kpc}$. This disk is made up of stellar and gaseous particles. Initially, the number of particles is $2 \times 10^{5}$, the disk mass is $M_{\text {disk }}^{\text {initial }}=4 \times 10^{10} M_{\odot}$, and its gas mass fraction is $30 \%$. The disk mass $M_{\text {disk }}$ and the number of gaseous particles increase with time because of gas accretion, as described in Sect. 2.5.

In 2-D, the composition and the evolution of the bulge and the halo are not studied. They are only represented as analytical potentials. They are implemented in our simulations as Plummer spheres. The associated potentials respectively read:

$\phi_{\text {bulge }}(r)=G \cdot M_{\mathrm{B}}\left(1+\frac{r^{2}}{a_{\mathrm{B}}^{2}}\right)^{-\frac{1}{2}}$

and

$\phi_{\text {halo }}(r)=G \cdot M_{\mathrm{H}}\left(1+\frac{r^{2}}{a_{\mathrm{H}}^{2}}\right)^{-\frac{1}{2}}$.

The bulge and halo masses, $M_{\mathrm{B}}$ and $M_{\mathrm{H}}$, are varied according to Table 1 . We here just note that $M_{\mathrm{B}}$ is generally smaller than
$M_{\text {disk}}$, and that the halo mass included in the disk radius, $M_{\text {halo }}^{\text {inner }}$, which is only a fraction of $M_{\mathrm{H}}$, is typically at most of the same order as $M_{\text {disk }}$. The $M_{\mathrm{B}} / M_{\text {disk }}^{\text {initial }}$ and $M_{\mathrm{H}} / M_{\text {disk }}^{\text {initial }}$ ratios are the two main parameters. On the other hand, the bulge and halo scale-lengths, $a_{\mathrm{B}}$ and $a_{\mathrm{H}}$, are fixed at $a_{\mathrm{B}}=1.4 \mathrm{kpc}$ and $a_{\mathrm{H}}=$ $25 \mathrm{kpc}$ (excepted in Sect. 3.3).

\subsection{Gravitational interaction and gas dissipation}

The gravitational potential is computed via Fourier transforms. The particles are meshed on a useful Cartesian grid of $512 \times 512$ cells, and each particle mass is assigned to four grid points according to the "cloud-in-cell" interpolation. Fourier transforms, carried out on a $1024 \times 1024$ grid in order to prevent Fourier periodic images do disturb the real disk, allow us to calculate the disk own potential, and derive forces. Then the analytical forces of the bulge and the halo are added, as they are described by (2) and (3). The potential differentiation and a bilinear interpolation give the gravitational force exerted on each particle. To suppress two-body relaxation, inefficient in real galaxies due to the large number of particles, the gravitational forces are softened with a Plummer softening, of characteristic size of $200 \mathrm{pc}$.

To represent the dissipative character of the gas component, a frequently used method is the sticky-particles algorithm, which schematizes the cloud-cloud collisions, and the non-elasticity of the collisions, where the relative kinetic energy is lost to heating the gas, which efficiently re-radiates it away (e.g. Combes \& Gerin 1985). The whole process tends to reduce the relative velocity of neighboring gas particles, cooling the gas through reducing its velocity dispersion. Here the process is represented by a dissipative force:

$\boldsymbol{f}=-k(\boldsymbol{v}-<\boldsymbol{v}>)$.

The local mean speed $<\boldsymbol{v}>$ is computed on a $1024 \times 1024$ grid. The dissipation time-scale $k^{-1}$ is chosen between 5 and $10 \mathrm{Gyr}$.

The code finally computes the integration of equations of motion with a leap-frog algorithm (Hut et al. 1995). The time step for the gravity potential computation, and for the integration of equations of motion, $\Delta t_{\phi}$, varies with time and is chosen to satisfy:

$\Delta t_{\phi}<\frac{L_{\text {cell }}}{V_{\max }}$

where $L_{\text {cell }}$ is the cell size, and $V_{\max }$ the maximum of the particles velocities. Computing $V_{\max }$ allows us to make $\Delta t_{\phi}$ vary proportionally to $L_{\text {cell }} / V_{\max }$. The time step then varies between 0.4 and 1 Myr. Then the effective number of times steps to reach the Hubble time is about 20000.

\subsection{Initial velocities}

While creating the initial disk, with a spatial distribution as described by (1), the code computes the mean circular velocity in each cell, the rotational frequency $\Omega$, the epicyclic frequency $\kappa$, etc. Random motions are given to all particles to prevent axisymmetric instabilities, their magnitude being at least equal to 
the critical speed (Toomre 1964). The Toomre parameter $Q$ is typically $Q=1.5$. The epicyclic approximation is used to relate the azimuthal and radial velocity dispersions:

$\sigma_{\theta}=\frac{\kappa}{2 \Omega} \sigma_{\mathrm{r}}$.

The rotational velocity is reduced from the circular speed, according to the Jeans equations, applied to the infinitely thin disk.

\subsection{Star formation and stellar mass-loss}

The star formation scheme and the way the stars lose mass to the gas are inspired from those described by Jungwiert et al. (2001). The stellar mass-loss is not approximated as instantaneous, but decreases with time as a power-law.

The star formation is assumed to be described by a local Schmidt law (Schmidt 1959), i.e. the star formation rate is proportional to a power of the surface density of gas $\mu_{\mathrm{gas}}^{N}$. We choose $N=1.4$, according to the observational determination of this parameter, on a global way and over large scales (see Kennicutt 1998a,b). The gas density is computed on a $1024 \times 1024$ grid, then the star formation rate is calculated for each cell of this grid. In each cell, the mass of young stars is taken away from all gas particles in the cell, in proportion to their mass at this epoch. This new star mass is added to all stellar particles present in the cell, in proportion to their mass. If there is no stellar particle in the cell, we wait a few time steps, until such a particle comes into the cell. This delays the actual star formation, yet, for $95 \%$ of the disk surface (the whole disk excepted its outer edge), such a situation occurs less than once 20 time steps: so, the delay in the star formation is generally avoided. When some mass is redistributed from a gas particle to a stellar one, the associated linear momentum is redistributed, too. When a mass $\Delta m$ is transferred from particle 1 (gas) to particle 2 (stellar), with initial masses $m_{1}$ and $m_{2}$ and initial velocities $\boldsymbol{v}_{1}$ and $\boldsymbol{v}_{2}$, the masses and velocities of particles are changed to:

$m_{1}^{\prime}=m_{1}-\Delta m$

$m_{2}^{\prime}=m_{2}+\Delta m$

$\boldsymbol{v}_{1}^{\prime}=\boldsymbol{v}_{1}$

$\boldsymbol{v}_{2}^{\prime}=\frac{m_{2} \boldsymbol{v}_{2}+\Delta m \boldsymbol{v}_{1}}{m_{2}^{\prime}}$.

This choice is not the only solution: the gas velocity is here conserved during star formation. One might also choose to add some velocity dispersion to new stars.

The number of particles is then conserved, but the particle masses vary. When a particle is accreted, it has the same mass as initially present gaseous and stellar particles. The mass of stellar particles doubles in about 15 Gyr, while gaseous particles particles lose about half of their initial mass in $10 \mathrm{Gyr}$.

Since each particle represents in fact already a cluster of actual components (star or gas cloud), the minimum mass of each particle being of the order of $10^{6} M_{\odot}$, the stellar mass-loss represents for each particle, the average mass-loss of an ensemble of stars, of various masses. As described in Jungwiert et al. (2001), we assume that a coeval stellar population has a massloss rate $\dot{M}_{\text {mass-loss }}(t)$ equal to:

$\dot{M}_{\text {mass-loss }}(t)=M_{\text {initial }} \frac{c_{0}}{t-t_{\mathrm{b}}+T_{0}}$

where $M_{\text {initial }}$ is the initial mass of the stellar population studied, and $t_{\mathrm{b}}$ its birth time. The two parameters are fixed to $c_{0}=5.35 \times 10^{-2}$ and $T_{0}=4.86 \mathrm{Myr}$.

However, such a mass-loss description is suitable only for a population of stars born in the same stellar cluster. We may use it for the initial particles, but it is not convenient for particles containing stars born at different epochs. Yet we have to add the mass of young stars in particles containing older ones in the above star formation scheme. Such additions may change the mass-loss rate of particles. Thus, we will define the massloss law of any particle in a recursive way:

- At $t=0$, any particle has a mass-loss rate given by (11);

- when new stars mass is added to a particle, we consider them as two merging particles: the old and young stellar particles. The first one is supposed to have a mass-loss rate given by:

$\dot{M}_{\text {old }}(t)=\frac{c_{\text {old }}}{t+T_{\text {old }}}$.

The second one contains a coeval population, therefore it has a mass-loss rate described by (11):

$\dot{M}_{\text {young }}(t)=\frac{c_{\text {young }}}{t+T_{\text {young }}}$.

After the merging, the new particle has a mass-loss rate that can be written:

$\dot{M}_{\text {new }}(t)=\frac{c_{\text {new }}}{t+T_{\text {new }}}$.

We just have to determine the parameters $c_{\text {new }}$ and $T_{\text {new }}$. We impose the continuity of the total stellar mass-loss rate at the instant $t$ of the fusion, which just reads:

$\frac{c_{\text {new }}}{t+T_{\text {new }}}=\frac{c_{\text {young }}}{t+T_{\text {young }}}+\frac{c_{\text {old }}}{t+T_{\text {old }}}$

and the conservation of the total stellar mass-loss integrated over 20 Gyr. These two equations determine the stellar mass-loss of a particle after the accretion of some young stars material.

Once the stellar mass-loss is computed, we distribute this mass over the neighboring gas particles. We use a $1024 \times 1024$ grid to compute the mass transfer in each cell. This mass is taken away from the star particles and transfered to the gas ones, in proportion to their mass, and the transfer of linear momentum that is associated with this mass redistribution is computed, just as was done for star formation.

These processes are not taken into account at the same frequency as the particle advance, and gravity potential computations. The time steps for stellar mass-loss and star formation are:

$\Delta t_{\mathrm{ML}}=\Delta t_{\mathrm{SF}}=4 \Delta t_{\phi}$ 


\subsection{Gas accretion}

To represent gas accretion in the two-dimensional code, some assumptions are necessary. We assume that the mean speed of accreted particles is equal to that of the outer disk, considering that slower particles have been accreted earlier, and that the accreted gas random velocity corresponds to the same Toomre parameter as the disk $(Q=1.5)$.

Accreted particles are placed just within the disk outer limit. None are put outside the disk. So, the disk radius is not directly changed in the process. The disk radius however increases indirectly, due to the disk response to accretion (see Sect. 3). The disk radius is then computed as a function of time, in order to continuously place new particles just below this radius, and not in the middle of the disk after some Gyr.

The accretion rate, $\dot{M}_{\text {accretion }}$, is chosen to make the disk mass double in 7 to $10 \mathrm{Gyr}$ (see for instance likely accretion rates, derived by Jiang \& Binney 1999). The gas is accreted from large-scale material. However, in some environments, it is possible that the accreted mass depletes a significant fraction of the halo mass. Yet, we will note that the existence or not of a halo mass depletion does not influence the disk evolution. We then generally consider that the accreted mass is partly taken away from the halo $\left(\dot{M}_{\text {halo }}=-\dot{M}_{\text {accret }} / 3\right)$, excepted in a few runs which are noticed in Table 1. Sect. 5 .

The consequences of all these assumptions are discussed in

\subsection{Three-dimensional code}

\subsubsection{Disk, gravitation, and star formation}

The 3-D model is quite similar to the 2-D one, as far as the spatial distributions projected on the plane are concerned. In addition the mass distribution of the initial disk in the $z$ direction is:

$\rho(r, z)=\sigma(r) \operatorname{sech}^{2}\left(\frac{z}{h_{0}}\right)$

where the disk scale height is $h_{0}=1.5 \mathrm{kpc}$, and $\sigma(r)$ is the disk surface density.

The gravitational potential is still obtained by an FFT method. We now use a useful grid of $128 \times 128 \times 128$. Proceeding Fourier transforms on a grid doubled in each dimension, as was done in the 2-D code, is not the best method as regards the computational efficiency. Instead we use the James (1977) method: appropriate masses are distributed on the grid boundaries to compensate for the Fourier periodic images.

Star formation and stellar mass-loss are included in this code with the same schemes as in the 2-D one.

\subsubsection{Sticky-particles code}

The two-dimensional gas dissipation scheme cannot really be used in three-dimensional simulations: computing the local mean speed in each cell implies that there are several gaseous particles in each cell. This would compel us either to use a few million particles, which means low computational efficiency, or to reduce the number of cells, which would make the spatial resolution reach a few kpc. Thus, we choose to use the sticky-particles algorithm (Schwarz 1981). When two gaseous particles collide, their radial (along the line joining them) and tangential relative velocities are reduced by factors $\beta_{\mathrm{r}}$ and $\beta_{\mathrm{t}}$. We choose $\beta_{\mathrm{r}}=0.65$ and $\beta_{\mathrm{t}}=0.65$ (see Jungwiert \& Palous 1996). Let us consider two particles at $\boldsymbol{r}_{1}$ and $\boldsymbol{r}_{2}$, with masses $m_{1}$ and $m_{2}$, and velocities $\boldsymbol{v}_{1}$ and $\boldsymbol{v}_{2}$ in the referential of their mass center. We note $M=m_{1}+m_{2}$, and:

$\boldsymbol{e}_{r}=\frac{\boldsymbol{r}_{2}-\boldsymbol{r}_{1}}{\left\|\boldsymbol{r}_{2}-\boldsymbol{r}_{1}\right\|}$

Before the collision, the radial et tangential relative velocities are respectively:

$\boldsymbol{v}_{\mathrm{r}}=\left(\left(\boldsymbol{v}_{2}-\boldsymbol{v}_{1}\right) \cdot \boldsymbol{e}_{\mathrm{r}}\right) \boldsymbol{e}_{\mathrm{r}}$

$v_{\mathrm{t}}=\left(\boldsymbol{v}_{2}-\boldsymbol{v}_{1}\right)-\left(\left(\boldsymbol{v}_{2}-\boldsymbol{v}_{1}\right) \cdot e_{\mathrm{r}}\right) e_{\mathrm{r}}$

After the collision, they are:

$\boldsymbol{v}_{\mathrm{r}}^{\prime}=\beta_{\mathrm{r}} \boldsymbol{v}_{\mathrm{r}}$

$\boldsymbol{v}_{\mathrm{t}}^{\prime}=\beta_{\mathrm{t}} \boldsymbol{v}_{\mathrm{t}}$

Then the new velocities of the particles in the referential of the mass center are:

$\boldsymbol{v}^{\prime}{ }_{1}=\frac{m_{2}}{M}\left(\beta_{\mathrm{t}}\left(\boldsymbol{v}_{1}-\boldsymbol{v}_{2}\right)+\left(\beta_{\mathrm{r}}-\beta_{\mathrm{t}}\right)\left(\left(\boldsymbol{v}_{1}-\boldsymbol{v}_{2}\right) \cdot \boldsymbol{e}_{\mathrm{r}}\right) \boldsymbol{e}_{\mathrm{r}}\right)$

$\boldsymbol{v}_{2}^{\prime}=\frac{m_{1}}{M}\left(\beta_{\mathrm{t}}\left(\boldsymbol{v}_{2}-\boldsymbol{v}_{1}\right)+\left(\beta_{\mathrm{r}}-\beta_{\mathrm{t}}\right)\left(\left(\boldsymbol{v}_{2}-\boldsymbol{v}_{1}\right) \cdot \boldsymbol{e}_{\mathrm{r}}\right) \boldsymbol{e}_{\mathrm{r}}\right)$.

The energy lost after the collision is then:

$\Delta E=\left(1-\beta_{\mathrm{r}}^{2}\right) \boldsymbol{v}_{\mathrm{r}}^{2}+\left(1-\beta_{\mathrm{t}}^{2}\right) \boldsymbol{v}_{\mathrm{t}}^{2}$.

We will discuss the influence of $\beta_{\mathrm{r}}$ and $\beta_{\mathrm{t}}$ on the results of our simulations in Appendix A.

\subsubsection{Gas accretion}

There are now many free parameters for gas accretion: the accreted gas angular momentum direction may be varied, and the accreted gas may be placed around any place of the disk, while in the 2-D code, it was always placed around the disk radius. We will precise which accretion parameters were used in each run when we study the 3-D results, in Sect. 4.

\section{Two-dimensional effects of gas accretion}

\subsection{General observations}

\subsubsection{Gas accretion and bar evolution}

Depending essentially on bulge to disk and halo to disk mass ratios, there are two kinds of bar evolution in 2-D simulations. We now describe them in turn. 
Table 1. Run parameters. The initial disk mass is always $M_{\text {disk }}^{\text {initial }}=4 \times 10^{10} M_{\odot}$. The runs F1 and F2 are introduced in Sect. 3.1. The runs A and B are studied in Sect. 3.2. Runs A1 to A9 and B1 to B7 are used to determine the dependence of bar evolution on parameters; in terms of bar evolution, these runs are rather similar either to run A or to run B. Runs R1a to R3c allow us to study the link with the de Vaucouleurs classification (see Sect. 3.3).

\begin{tabular}{|c|c|c|c|c|c|c|c|}
\hline Run & $a_{\text {disk }}$ & $a_{\text {halo }}$ & $M_{\text {halo }}^{\text {inner }}$ & $M_{\text {bulge }}$ & $\dot{M}_{\text {accret }}$ & $\dot{M}_{\text {halo }}$ & initial gas mass rate \\
\hline $\mathrm{F} 1$ & $4 \mathrm{kpc}$ & $25 \mathrm{kpc}$ & $2.5 \times 10^{10} M_{\odot}$ & $1.3 \times 10^{10} M_{\odot}$ & $0.6 \times 10^{10} M_{\odot} \mathrm{Gyr}^{-1}$ & $-\dot{M}_{\text {accret }} / 2$ & $25 \%$ \\
\hline 0F1 & $4 \mathrm{kpc}$ & $25 \mathrm{kpc}$ & $2.5 \times 10^{10} M_{\odot}$ & $1.3 \times 10^{10} M_{\odot}$ & 0 & $-\dot{M}_{\text {accret }} / 2$ & $25 \%$ \\
\hline $\mathrm{F} 2$ & $4 \mathrm{kpc}$ & $25 \mathrm{kpc}$ & $4.0 \times 10^{10} M_{\odot}$ & $4.0 \times 10^{10} M_{\odot}$ & $0.6 \times 10^{10} M_{\odot} \mathrm{Gyr}^{-1}$ & $-\dot{M}_{\text {accret }} / 2$ & $25 \%$ \\
\hline $0 \mathrm{~F} 2$ & $4 \mathrm{kpc}$ & $25 \mathrm{kpc}$ & $4.0 \times 10^{10} M_{\odot}$ & $4.0 \times 10^{10} M_{\odot}$ & 0 & $-\dot{M}_{\text {accret }} / 2$ & $25 \%$ \\
\hline A & $4 \mathrm{kpc}$ & $25 \mathrm{kpc}$ & $2.5 \times 10^{10} M_{\odot}$ & $3.0 \times 10^{10} M_{\odot}$ & $0.57 \times 10^{10} M_{\odot} \mathrm{Gyr}^{-1}$ & $-\dot{M}_{\text {accret }} / 3$ & $20 \%$ \\
\hline $0 \mathrm{~A}$ & $4 \mathrm{kpc}$ & $25 \mathrm{kpc}$ & $2.5 \times 10^{10} M_{\odot}$ & $3.0 \times 10^{10} M_{\odot}$ & 0 & $-\dot{M}_{\text {accret }} / 3$ & $20 \%$ \\
\hline B & $4 \mathrm{kpc}$ & $25 \mathrm{kpc}$ & $3.510^{10} \mathrm{M}_{\odot}$ & $1.410^{10} \mathrm{M}_{\odot}$ & $0.57 \times 10^{10} M_{\odot} \mathrm{Gyr}^{-1}$ & $-\dot{M}_{\text {accret }} / 3$ & $20 \%$ \\
\hline OB & $4 \mathrm{kpc}$ & $25 \mathrm{kpc}$ & $3.5 \times 10^{10} M_{\odot}$ & $1.4 \times 10^{10} M_{\odot}$ & 0 & $-\dot{M}_{\text {accret }} / 3$ & $20 \%$ \\
\hline A 1 & $4 \mathrm{kpc}$ & $25 \mathrm{kpc}$ & $2.0 \times 10^{10} M_{\odot}$ & $1.7 \times 10^{10} M_{\odot}$ & $0.5 \times 10^{10} M_{\odot} \mathrm{Gyr}^{-1}$ & $-\dot{M}_{\text {accret }}$ & $20 \%$ \\
\hline Alh & $4 \mathrm{kpc}$ & $25 \mathrm{kpc}$ & $2.0 \times 10^{10} M_{\odot}$ & $1.7 \times 10^{10} M_{\odot}$ & $0.5 \times 10^{10} M_{\odot} \mathrm{Gyr}^{-1}$ & 0 & $20 \%$ \\
\hline $\mathrm{A} 2$ & $4 \mathrm{kpc}$ & $25 \mathrm{kpc}$ & $2.0 \times 10^{10} M_{\odot}$ & $2.5 \times 10^{10} M_{\odot}$ & $0.5 \times 10^{10} M_{\odot} \mathrm{Gyr}^{-1}$ & $-\dot{M}_{\text {accret }} / 3$ & $20 \%$ \\
\hline A3 & $4 \mathrm{kpc}$ & $25 \mathrm{kpc}$ & $2.0 \times 10^{10} M_{\odot}$ & $3.5 \times 10^{10} M_{\odot}$ & $0.5 \times 10^{10} M_{\odot} \mathrm{Gyr}^{-1}$ & $-\dot{M}_{\text {accret }} / 3$ & $20 \%$ \\
\hline A4 & $4 \mathrm{kpc}$ & $25 \mathrm{kpc}$ & $3.0 \times 10^{10} M_{\odot}$ & $2.5 \times 10^{10} M_{\odot}$ & $0.5 \times 10^{10} M_{\odot} \mathrm{Gyr}^{-1}$ & $-\dot{M}_{\text {accret }}$ & $20 \%$ \\
\hline A4h & $4 \mathrm{kpc}$ & $25 \mathrm{kpc}$ & $3.0 \times 10^{10} M_{\odot}$ & $2.5 \times 10^{10} M_{\odot}$ & $0.5 \times 10^{10} M_{\odot} \mathrm{Gyr}^{-1}$ & 0 & $20 \%$ \\
\hline A5 & $4 \mathrm{kpc}$ & $25 \mathrm{kpc}$ & $3.0 \times 10^{10} M_{\odot}$ & $3.5 \times 10^{10} M_{\odot}$ & $0.5 \times 10^{10} M_{\odot} \mathrm{Gyr}^{-1}$ & $-\dot{M}_{\text {accret }} / 3$ & $20 \%$ \\
\hline A6 & $4 \mathrm{kpc}$ & $25 \mathrm{kpc}$ & $4.5 \times 10^{10} M_{\odot}$ & $2.5 \times 10^{10} M_{\odot}$ & $0.5 \times 10^{10} M_{\odot} \mathrm{Gyr}^{-1}$ & $-\dot{M}_{\text {accret }} / 3$ & $20 \%$ \\
\hline A7 & $4 \mathrm{kpc}$ & $25 \mathrm{kpc}$ & $4.5 \times 10^{10} M_{\odot}$ & $3.5 \times 10^{10} M_{\odot}$ & $0.5 \times 10^{10} M_{\odot} \mathrm{Gyr}^{-1}$ & $-\dot{M}_{\text {accret }} / 3$ & $20 \%$ \\
\hline A8 & $4 \mathrm{kpc}$ & $25 \mathrm{kpc}$ & $5.5 \times 10^{10} M_{\odot}$ & $2.5 \times 10^{10} M_{\odot}$ & $0.5 \times 10^{10} M_{\odot} \mathrm{Gyr}^{-1}$ & $-\dot{M}_{\text {accret }}$ & $20 \%$ \\
\hline $\mathrm{A} 8 \mathrm{~h}$ & $4 \mathrm{kpc}$ & $25 \mathrm{kpc}$ & $5.5 \times 10^{10} M_{\odot}$ & $2.5 \times 10^{10} M_{\odot}$ & $0.5 \times 10^{10} M_{\odot} \mathrm{Gyr}^{-1}$ & 0 & $20 \%$ \\
\hline A9 & $4 \mathrm{kpc}$ & $25 \mathrm{kpc}$ & $5.5 \times 10^{10} M_{\odot}$ & $3.5 \times 10^{10} M_{\odot}$ & $0.5 \times 10^{10} M_{\odot} \mathrm{Gyr}^{-1}$ & $-\dot{M}_{\text {accret }} / 3$ & $20 \%$ \\
\hline B1 & $4 \mathrm{kpc}$ & $25 \mathrm{kpc}$ & $2.0 \times 10^{10} M_{\odot}$ & $1.0 \times 10^{10} M_{\odot}$ & $0.5 \times 10^{10} M_{\odot} \mathrm{Gyr}^{-1}$ & $-\dot{M}_{\text {accret }} / 3$ & $20 \%$ \\
\hline B2 & $4 \mathrm{kpc}$ & $25 \mathrm{kpc}$ & $3.0 \times 10^{10} M_{\odot}$ & $1.0 \times 10^{10} M_{\odot}$ & $0.5 \times 10^{10} M_{\odot} \mathrm{Gyr}^{-1}$ & $-\dot{M}_{\text {accret }} / 3$ & $20 \%$ \\
\hline B3 & $4 \mathrm{kpc}$ & $25 \mathrm{kpc}$ & $4.0 \times 10^{10} M_{\odot}$ & $1.7 \times 10^{10} M_{\odot}$ & $0.5 \times 10^{10} M_{\odot} \mathrm{Gyr}^{-1}$ & $-\dot{M}_{\text {accret }} / 3$ & $20 \%$ \\
\hline B4 & $4 \mathrm{kpc}$ & $25 \mathrm{kpc}$ & $4.5 \times 10^{10} M_{\odot}$ & $1.0 \times 10^{10} M_{\odot}$ & $0.5 \times 10^{10} M_{\odot} \mathrm{Gyr}^{-1}$ & $-\dot{M}_{\text {accret }} / 3$ & $20 \%$ \\
\hline B5 & $4 \mathrm{kpc}$ & $25 \mathrm{kpc}$ & $4.5 \times 10^{10} M_{\odot}$ & $1.7 \times 10^{10} M_{\odot}$ & $0.5 \times 10^{10} M_{\odot} \mathrm{Gyr}^{-1}$ & $-\dot{M}_{\text {accret }}$ & $20 \%$ \\
\hline B5h & $4 \mathrm{kpc}$ & $25 \mathrm{kpc}$ & $4.5 \times 10^{10} M_{\odot}$ & $1.7 \times 10^{10} M_{\odot}$ & $0.5 \times 10^{10} M_{\odot} \mathrm{Gyr}^{-1}$ & 0 & $20 \%$ \\
\hline B6 & $4 \mathrm{kpc}$ & $25 \mathrm{kpc}$ & $5.5 \times 10^{10} M_{\odot}$ & $1.0 \times 10^{10} M_{\odot}$ & $0.5 \times 10^{10} M_{\odot} \mathrm{Gyr}^{-1}$ & $-\dot{M}_{\text {accret }} / 3$ & $20 \%$ \\
\hline B7 & $4 \mathrm{kpc}$ & $25 \mathrm{kpc}$ & $5.5 \times 10^{10} M_{\odot}$ & $1.7 \times 10^{10} M_{\odot}$ & $0.5 \times 10^{10} M_{\odot} \mathrm{Gyr}^{-1}$ & $-\dot{M}_{\text {accret }} / 3$ & $20 \%$ \\
\hline R1a & $4 \mathrm{kpc}$ & $25 \mathrm{kpc}$ & $2.5 \times 10^{10} M_{\odot}$ & $1.5 \times 10^{10} M_{\odot}$ & $0.6 \times 10^{10} M_{\odot} \mathrm{Gyr}^{-1}$ & $-\dot{M}_{\text {accret }} / 3$ & $20 \%$ \\
\hline $\mathrm{R} 1 \mathrm{~b}$ & $4 \mathrm{kpc}$ & $25 \mathrm{kpc}$ & $4.0 \times 10^{10} M_{\odot}$ & $2.5 \times 10^{10} M_{\odot}$ & $0.6 \times 10^{10} M_{\odot} \mathrm{Gyr}^{-1}$ & $-\dot{M}_{\text {accret }} / 3$ & $20 \%$ \\
\hline $\mathrm{R} 1 \mathrm{c}$ & $4 \mathrm{kpc}$ & $25 \mathrm{kpc}$ & $5.5 \times 10^{10} M_{\odot}$ & $3.5 \times 10^{10} M_{\odot}$ & $0.6 \times 10^{10} M_{\odot} \mathrm{Gyr}^{-1}$ & $-\dot{M}_{\text {accret }} / 3$ & $20 \%$ \\
\hline $\mathrm{R} 2 \mathrm{a}$ & $5 \mathrm{kpc}$ & $20 \mathrm{kpc}$ & $2.5 \times 10^{10} M_{\odot}$ & $1.5 \times 10^{10} M_{\odot}$ & $0.6 \times 10^{10} M_{\odot} \mathrm{Gyr}^{-1}$ & $-\dot{M}_{\text {accret }} / 3$ & $20 \%$ \\
\hline $\mathrm{R} 2 \mathrm{~b}$ & $5 \mathrm{kpc}$ & $20 \mathrm{kpc}$ & $4.0 \times 10^{10} M_{\odot}$ & $2.5 \times 10^{10} M_{\odot}$ & $0.6 \times 10^{10} M_{\odot} \mathrm{Gyr}^{-1}$ & $-\dot{M}_{\text {accret }} / 3$ & $20 \%$ \\
\hline $\mathrm{R} 2 \mathrm{c}$ & $5 \mathrm{kpc}$ & $20 \mathrm{kpc}$ & $5.5 \times 10^{10} M_{\odot}$ & $3.5 \times 10^{10} M_{\odot}$ & $0.6 \times 10^{10} M_{\odot} \mathrm{Gyr}^{-1}$ & $-\dot{M}_{\text {accret }} / 3$ & $20 \%$ \\
\hline $\mathrm{R} 3 \mathrm{a}$ & $6 \mathrm{kpc}$ & $15 \mathrm{kpc}$ & $2.5 \times 10^{10} M_{\odot}$ & $1.5 \times 10^{10} M_{\odot}$ & $0.6 \times 10^{10} M_{\odot} \mathrm{Gyr}^{-1}$ & $-\dot{M}_{\text {accret }} / 3$ & $20 \%$ \\
\hline $\mathrm{R} 3 \mathrm{~b}$ & $6 \mathrm{kpc}$ & $15 \mathrm{kpc}$ & $4.0 \times 10^{10} M_{\odot}$ & $2.5 \times 10^{10} M_{\odot}$ & $0.6 \times 10^{10} M_{\odot} \mathrm{Gyr}^{-1}$ & $-\dot{M}_{\text {accret }} / 3$ & $20 \%$ \\
\hline $\mathrm{R} 3 \mathrm{c}$ & $6 \mathrm{kpc}$ & $15 \mathrm{kpc}$ & $5.5 \times 10^{10} M_{\odot}$ & $3.5 \times 10^{10} M_{\odot}$ & $0.6 \times 10^{10} M_{\odot} \mathrm{Gyr}^{-1}$ & $-\dot{M}_{\text {accret }} / 3$ & $20 \%$ \\
\hline G1 & $4 \mathrm{kpc}$ & $25 \mathrm{kpc}$ & $2.5 \times 10^{10} M_{\odot}$ & $2.0 \times 10^{10} M_{\odot}$ & $0.5 \times 10^{10} M_{\odot} \mathrm{Gyr}^{-1}$ & $-\dot{M}_{\text {accret }} / 3$ & $5 \%$ \\
\hline G2 & $4 \mathrm{kpc}$ & $25 \mathrm{kpc}$ & $2.5 \times 10^{10} M_{\odot}$ & $2.0 \times 10^{10} M_{\odot}$ & $0.5 \times 10^{10} M_{\odot} \mathrm{Gyr}^{-1}$ & $-\dot{M}_{\text {accret }} / 3$ & $15 \%$ \\
\hline G3 & $4 \mathrm{kpc}$ & $25 \mathrm{kpc}$ & $5.5 \times 10^{10} M_{\odot}$ & $2.0 \times 10^{10} M_{\odot}$ & $0.5 \times 10^{10} M_{\odot} \mathrm{Gyr}^{-1}$ & $-\dot{M}_{\text {accret }} / 3$ & $25 \%$ \\
\hline G4 & $4 \mathrm{kpc}$ & $25 \mathrm{kpc}$ & $2.5 \times 10^{10} M_{\odot}$ & $2.0 \times 10^{10} M_{\odot}$ & $0.5 \times 10^{10} M_{\odot} \mathrm{Gyr}^{-1}$ & $-\dot{M}_{\text {accret }} / 3$ & $40 \%$ \\
\hline
\end{tabular}



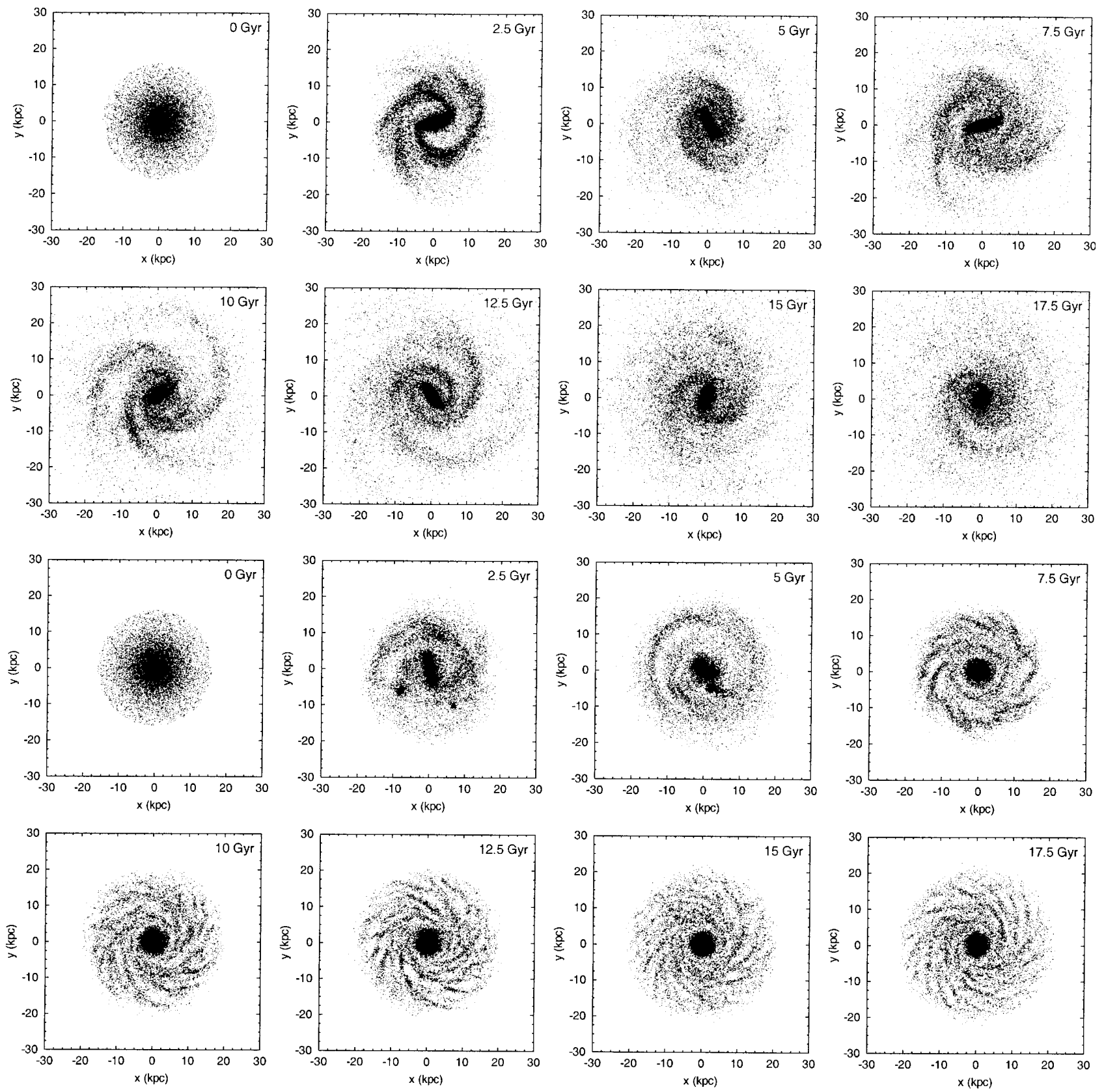

Fig. 1. Runs F1 and $0 \mathrm{~F} 1$ : disk evolution with accretion and bar maintenance (F1, top), and without accretion (0F1, bottom). These plots of mass distribution respect the gas-to-star mass ratio. One particle on twenty is represented.

Bar maintenance Let us first examine the model of run F1, the parameters of which are given in Table 1 . The disk evolution is plotted in Fig. 1.

Without gas accretion, this disk develops a bar, with a shortlifetime. The bar is very strong around 1 and $2 \mathrm{Gyr}$. The central condensation rises very fast, and destroys the bar in less than 7 Gyr. The introduction of gas accretion makes the bar more long-lived. The disk has a bar wave during more than 15 Gyr. The bar destruction process still occurs, but is much slower. Therefore, one of the main effects of gas accretion is to maintain bars in such galactic disks.

Moreover, the maintained bar can still be strong enough to correspond to a morphological SB-type, after more than
10 Gyr. A SB-type disk or strongly barred disk is a disk in which the bar is very long, with two arms connected to the bar. In a SAB-type disk, the bar is less long and has rather a lens aspect; the spiral structure is rather disconnected from the bar.

A strong bar is able to influence the spiral pattern outside of its corotation, so that the spiral and the bar have the same pattern speed. In the run F1, at $15 \mathrm{Gyr}$, the spiral pattern appears always in phase with the bar, as in SB-type galaxies. Even if the bar is stronger during the first Gyrs, the disk may become more weakly barred, as an SAB-type, and return to SB-type with an older bar. The frequencies of SB and SAB-type disks are studied in Sect. 3.3. 

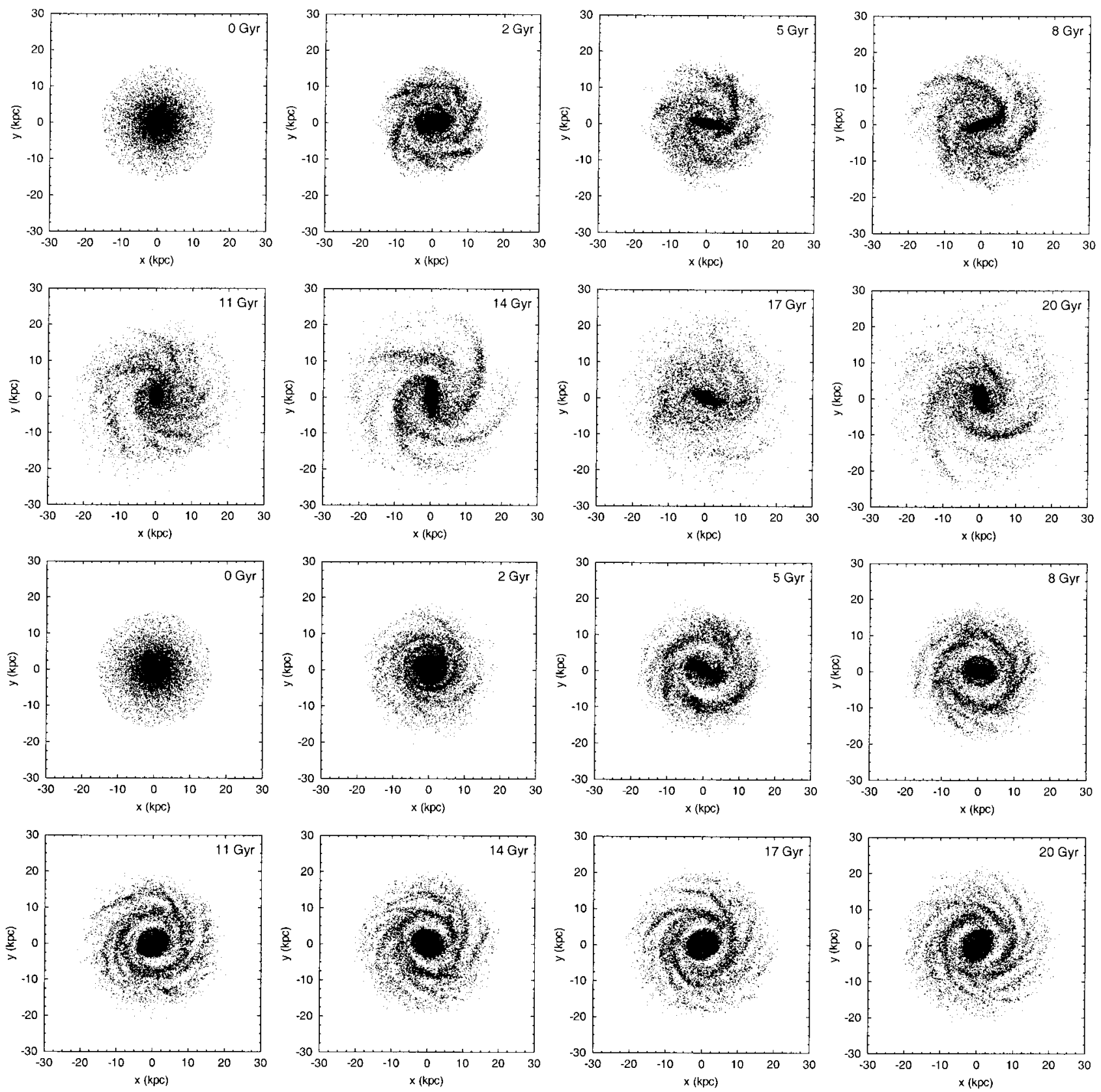

Fig. 2. Runs F2 and OF2: disk evolution with accretion and bar destruction and re-formation (F2, top), and without accretion (0F2, bottom). Here, the bar is totally destroyed before its re-formation. It can also be much weakened without disappearing (see Fig. 4 for such a situation in model A). Two bar episodes can be seen here in 20 Gyrs; three or four bar episodes have been followed as well in similar runs (A1-A9).

Runs B and B1 to B7 also show such a bar maintenance. The bar lifetime for each of them is described in Table 2.

Bar destruction and re-formation. The second situation, obtained for instance with parameters of run F2 from Table 1, corresponds to bar destruction and re-formation. The disk evolution of this run is shown in Fig. 2.

Now, the isolated disk has a strong bar during less than 2 Gyr. This bar destroys itself very fast. Then, a small lensshaped bar remains in the inner disk, which testifies a previous strong bar existence. Even if the real bar is a short life-time one, the lens is observed over more than $10 \mathrm{Gyr}$.
Gas accretion allows the bar to reappear. After being totally unbarred - even the lens is now destructed -, the disks undergoes a new bar episode. A cycle of bar destruction and re-formation takes places in such disks. We generally obtain 2 or 3 cycles in $20 \mathrm{Gyr}$, sometimes 4 .

If the strength of the bar tends to decrease with time, because of the mass concentration, and the disk heating (larger velocity dispersion), there still can exist quite strong bars (SB-types) after 10 Gyr. Due to gas accretion, and consequent disk cooling, the second or the third bars can be strong, almost as strong as the first bar. 
Table 2. Bar lifetime for bar maintaining galaxies.

\begin{tabular}{lc}
\hline \hline Run & Bar lifetime \\
\hline B1 & 13.5 \\
B2 & 15 \\
B3 & 17 \\
B4 & 12.5 \\
B5 & 19 \\
B5h & 18.5 \\
B6 & 13 \\
B7 & 15.5 \\
\hline
\end{tabular}

Table 3. Number of successive bars followed over 20 Gyrs in bar re-forming galaxies.

\begin{tabular}{lc}
\hline \hline Run & Number of bars in 20 Gyrs \\
\hline A1 & 4 \\
A1h & 4 \\
A2 & 2 \\
A3 & 2 \\
A4 & 3 \\
A4h & 3 \\
A5 & 2 \\
A6 & 3 \\
A7 & 2 \\
A8 & 3 \\
A8h & 3 \\
A9 & 2 \\
\hline
\end{tabular}

In runs $\mathrm{A}$ and $\mathrm{A} 1$ to $\mathrm{A} 9$, we also follow such bar destructions and re-formations. The number of bars followed over 20 Gyrs for these runs is given in Table 3.

Dependence of bar evolution on parameters. A galactic disk may either keep a single bar or get several successive bars. By simulating several disks, we study the dependence on physical parameters. We find that the two determining parameters are the bulge to disk and halo to disk masses ratios. Light bulge galaxies maintain their bar, while bars are destructed and regenerated in heavy bulge galaxies. The halo mass has also an effect on the disk evolution. The bar evolution of a galactic disk, depending on bulge and halo to disk masses ratios, is shown in Fig. 3.

The bulge mass also influences the speed of the destruction and re-formation cycle, in bar re-forming galaxies. It influences the bar lifetime in bar maintaining galaxies. Both influences are explained in Fig. 3.

The initial gas to stellar disk mass ratio has also been varied (runs G1-G4). The higher it is, the stronger the density waves are during the 1 or 2 first Gyr. Yet, this ratio does not really influence the long-term evolution of the disk: after a few Gyr, the gas to stellar mass ratio depends more on the accretion rate rather than on the initial gas to stellar mass ratio.

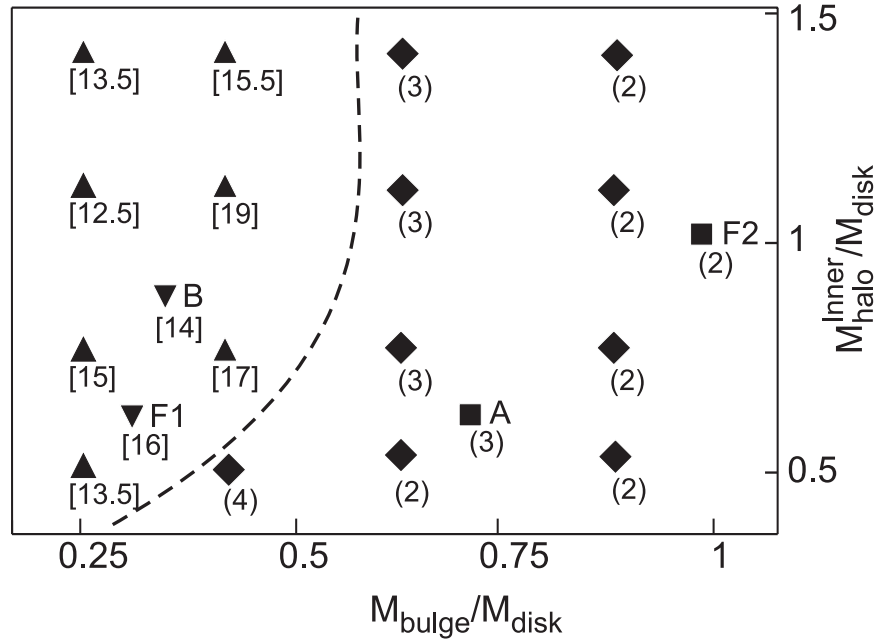

$\Delta$ Runs B1-B7: Bar maintenance. [bar lifetime in Gyr]

Runs A1-A9: Bar destruction and re-formation. (number of bars over $20 \mathrm{Gyr}$ )

Fig. 3. Disk evolution depending on bulge to disk and halo to disk masses ratio. $M_{\text {disk }}$ is the initial disk mass, and $M_{\text {halo }}^{\text {inner }}$ is one tenth $M_{\text {halo }}$.

\subsubsection{Other consequences of accretion}

The main other consequences of accretion are:

- the spiral structure is rejuvenated by accretion (see also Sect. 3.3). Arms have roughly the same morphology around $15 \mathrm{Gyr}$ as around $3 \mathrm{Gyr}$, whereas in non accreting disks, they become weaker, since the gas density then decreases in the disk while the gas accumulates in an inner ring and/or an outer ring;

- the disk radius increases; accreted gas, even if always placed inside the disk border, produces larger disks;

- there is sometimes a gaseous outer ring, which is stronger than without accretion. This, as shown below, is due to the accreted gas repelled outside the corotation because of bar torques;

- the bar pattern speed raises; this will be shown and interpreted in Sect. 3.2.

The three last phenomena are now studied and explained.

\subsection{Detailed study of two galactic disks}

Let us now present two runs that have been studied in detail, runs $\mathrm{A}$ and $\mathrm{B}$ in Table 1.

Model A corresponds to a disk which undergoes three successive bars, whereas model B corresponds to a single bar that persists over a Hubble time. The bar strength evolutions for both models are shown in Fig. 4. The bar strength is defined as the ratio of the tangential force of the $m=2$ Fourier component, normalized to the radial force. If the potential is decomposed as

$\Phi(r, \theta)=\Phi_{0}(r)+\sum_{m} \Phi_{m}(r) \cos \left(m \theta-\phi_{m}\right)$ 

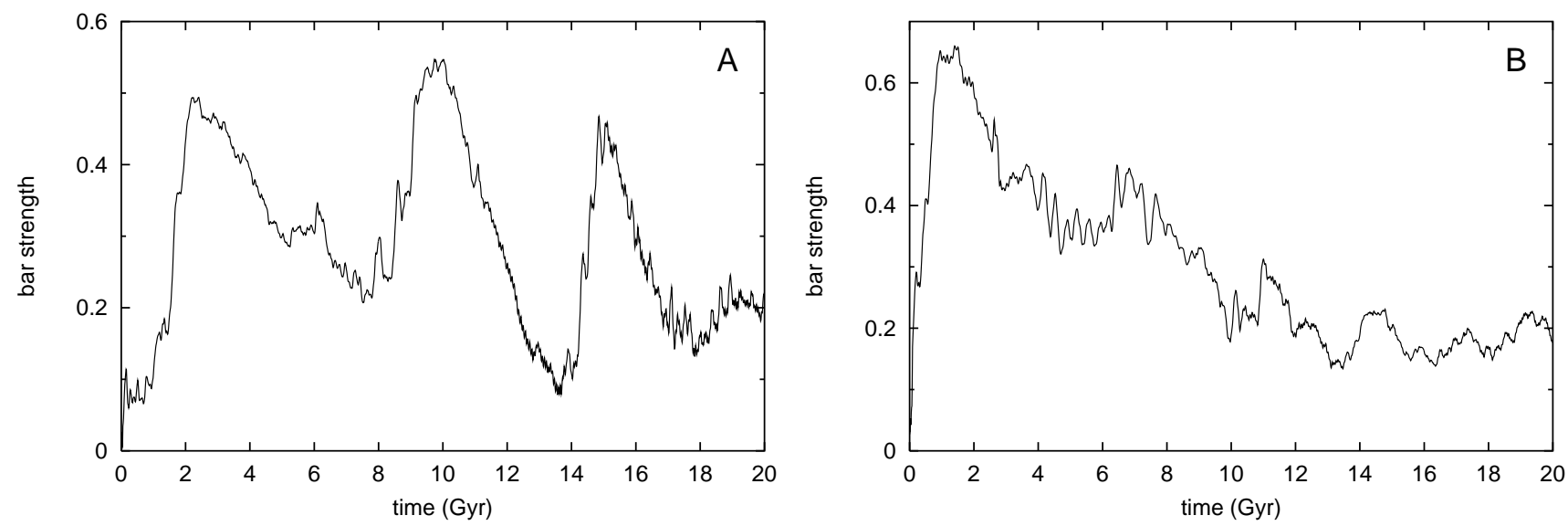

Fig. 4. Bar strength evolution in models A (left) and B (right).
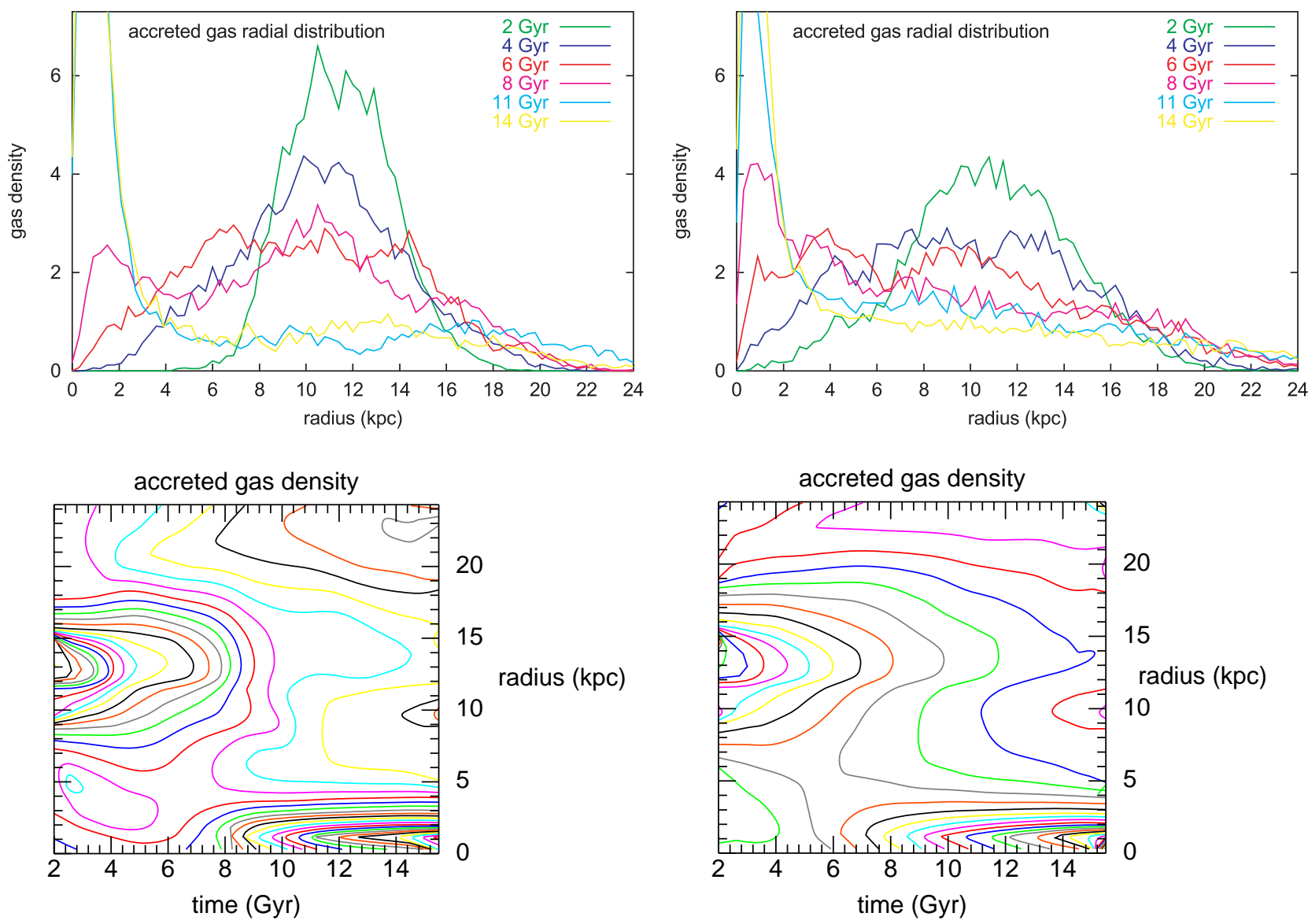

Fig. 5. Evolution of the radial distribution of accreted during the first 1.5 Gyr gas. Model A is on the left, model B is on the right.

the bar strength is the maximum over radius of

$S_{2}=2 \Phi_{2} / r\left|F_{\mathrm{r}}\right|$

where $F_{\mathrm{r}}=-\partial \Phi_{0} / \partial r$.

The cause of these two different scenarii in models A and $\mathrm{B}$, the evolution of the bar pattern speed, and the increase in the disk radius, are now developed and studied.

\subsubsection{Evolution of the accreted gas distribution}

The accreted gas is first located in the outer disk. Yet, non axisymmetric gravity fields and dissipation make its distribution evolve. The accreted gas distribution and its evolution are shown in Fig. 5. Only particles that are accreted during the first $1.5 \mathrm{Gyr}$ are taken into account in this figure, in order to have a reasonable time axis, as the accretion period of such particles is rather short in comparison with the galaxy evolution time. 

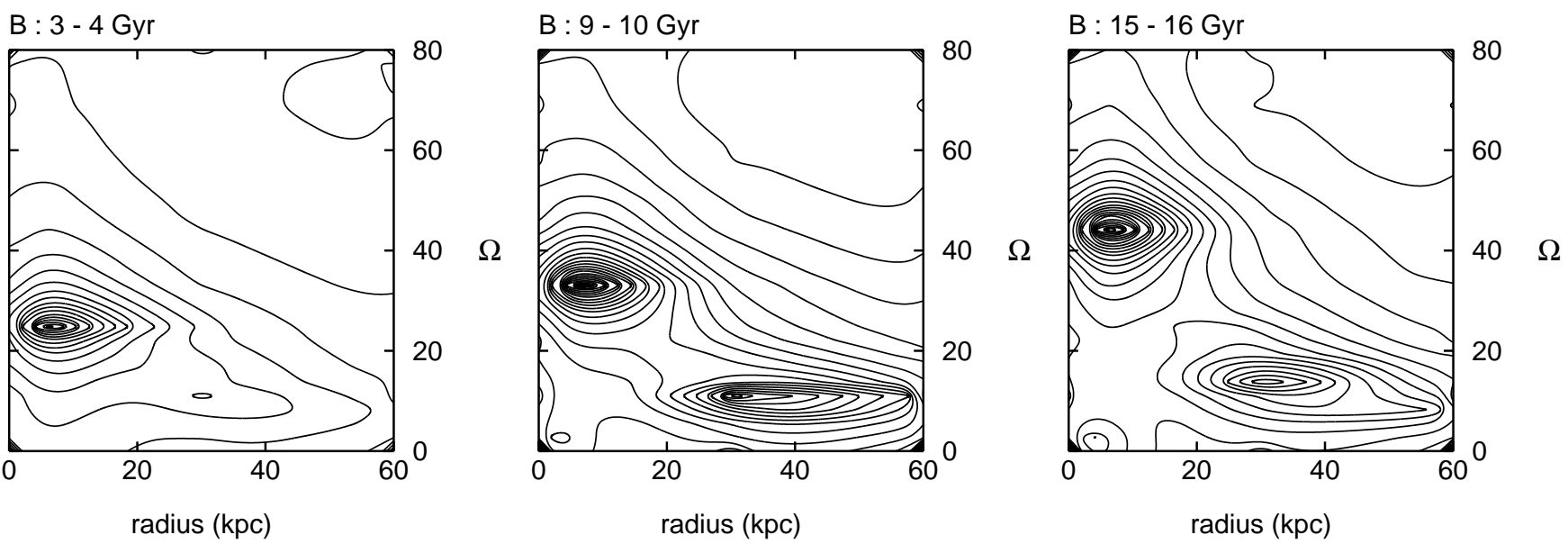

Fig. 6. Second harmonic of the potential, as a function of radius and pulsation, for model B. The main maximum (at small radii and high frequencies) corresponds to the bar, the secondary one (at large radii and low frequencies) to spiral waves. Similar analysis are done for model A.

If we consider the particles that are accreted between any epoch $t$ and $t+1.5 \mathrm{Gyr}$, the results are very similar to those plotted in Fig. 5.

For both models $\mathrm{A}$ and $\mathrm{B}$, we see that the accreted gas density is never high in the region between 4 and $7 \mathrm{kpc}$, which corresponds to the bar corotation in both models. Tangential forces exerted by the bar prevent gas from staying in this part of the disk. Then, accreted particles may either stay in the outer disk, or fall towards the disk center, if some dissipation make them go there. Actually, we see in Fig. 5 that accreted gas accumulates around the galactic center after some Gyr. To go there, it must overcome bar positive torques. The only mechanism responsible is the gas dissipation, which becomes higher and higher as gas density outside the corotation increases. By spreading out in the disk, some gas reaches the corotation. Then, bar negative torques drive it quickly into the disk center.

Yet, there are some differences between models A and B. The gas repelled outside the corotation seems to be more abundant for model A. The particles get over the corotation later, after 8 Gyr instead of 6 or 7 Gyr for model B. This interval is rather small, but it is significant that for model $\mathrm{B}$, there is still a strong bar when the gas falls inside the corotation, whereas in model $\mathrm{A}$, the corotation crossing occurs only when the bar strength is minimum. Moreover, the gas infall inside the corotation is rather regular and takes some Gyrs for model B; for model A this infall is faster and happens at once, between the two first bar episodes.

This difference concerning the accreted gas repartition allows us to account for the two encountered situations. Moreover, it will explain that, in the next subsections, different phenomena occur in a continuous way for model $\mathrm{B}$, and only between bar episodes for model A.

\subsubsection{Explanation of bar destruction and re-formation or maintenance}

The common point between models $\mathrm{A}$ and $\mathrm{B}$ is that gas accretion favours bars. The reason for that is the increase in the disk mass. In non accreting disks, the central condensation mass raises, which stabilizes the disk against barred waves. Accretion makes the disk mass increase and become more selfgravitating, while making the central (inner disk and bulge) become relatively lower, which may either prevent the bar from being destroyed or re-form a bar.

The difference takes place in the accreted gas repartition. For model B, this gas falls inside the corotation continuously. So, the region of the possible bar (which is $r<4$ to $6 \mathrm{kpc}$ ) increases in weight regularly. The bar is then favored regularly, and is maintained a long time.

For model A, the effects favourable to bars occur only between the bar episodes. During such a bar episode, the accreted gas stays in the outer disk. Even if the total disk mass rises, only the outer regions increase in mass: this cannot maintain the bar nor create a new one. Between two bar episodes, the region of the possible bar gains mass. The possible barred region to central condensation mass ratio becomes lower, and a new bar wave appears.

Let us consider now the bar pattern speed evolution, and how it is related to the accreted gas distribution.

\subsubsection{Influence of accretion on bar pattern speed}

The bar pattern speed, $\Omega_{\mathrm{b}}$, has been measured via temporal Fourier transform of the $m=2$ harmonic of the potential. Figure 6 shows the intensity of this harmonic as a function of radius and frequency $\Omega$, around 3.5, 9.5 and 15.5 Gyr. Using such Fourier analysis, we are able to summarize $\Omega_{\mathrm{b}}$ as a function of time, as shown in Fig. 7.

This reveals a significant increase in the bar pattern speed, which may double over a Hubble time. The difference between the two A and B cases is that this growth is either smooth or irregular. This suggests that the bar acceleration is closely linked to gas accretion, since it has the same evolution as the accreted gas distribution in both models $\mathrm{A}$ and $\mathrm{B}$.

The implications of such a pattern speed increase are developed below. Let us first analyze the reasons for this phenomenon. 


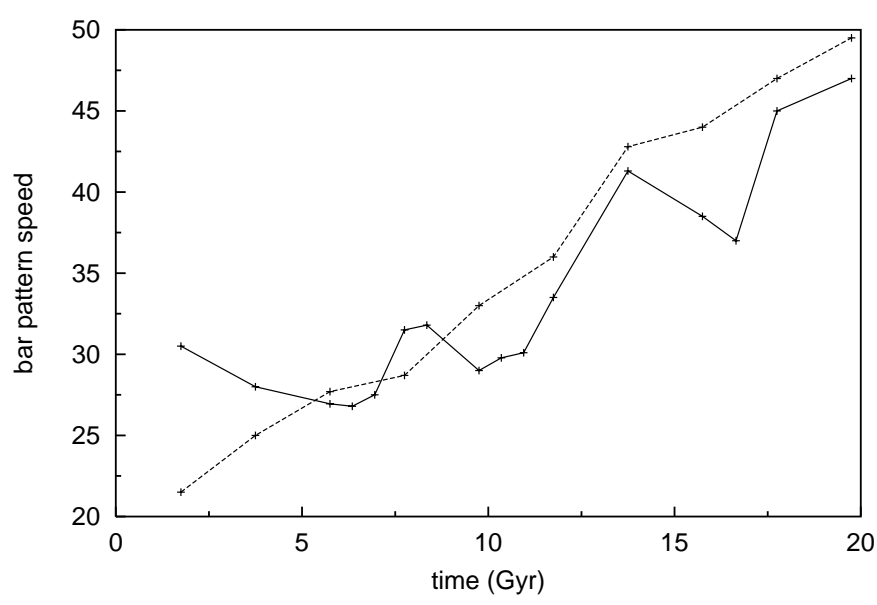

Fig. 7. Bar pattern speed as a function of time, for model A (full line) and model B (dashed line).

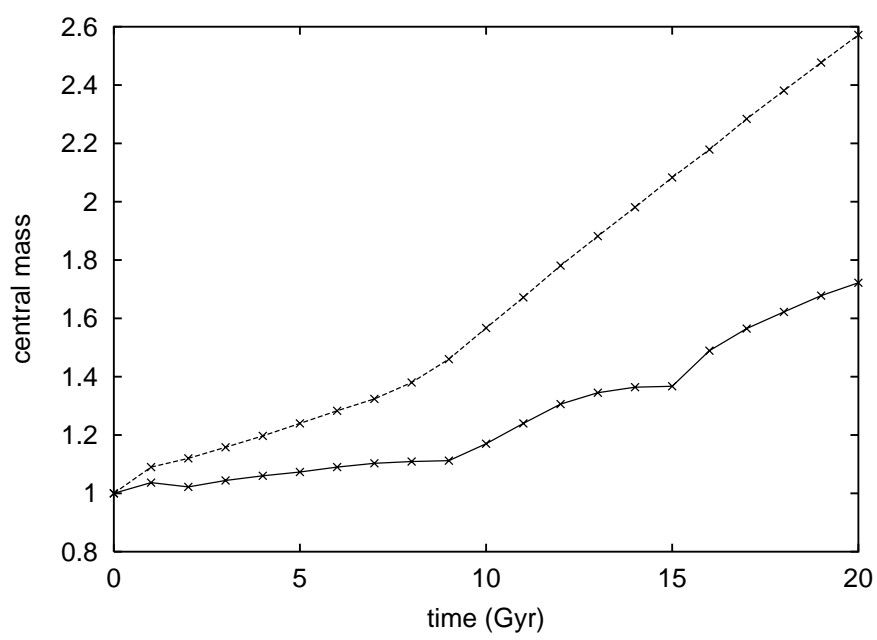

Fig. 8. Evolution of the disk inner mass, $M_{\text {disk }}(2 \mathrm{kpc})$, normalized to its initial value, for model A (full line) and model B (dashed line). Bulge and halo inner masses are to be added to it to give $M_{\text {inner }}$.

Table 4. Inner mass and bar pattern speed growth rates (model A).

\begin{tabular}{ccc}
\hline \hline growth factor between the first bar and... & $\sqrt{M_{\text {inner }}}$ & $\Omega_{\mathrm{b}}$ \\
\hline ...the second bar & 1.06 & 1.11 \\
\hline ...the third bar & 1.20 & 1.37 \\
\hline
\end{tabular}

\subsubsection{Physical cause of the bar acceleration}

Central condensation growth A possible cause of the growth in the bar pattern speed is the inner mass accumulation. For every particles, this would make $\Omega$ and $\kappa$ rise. If we write $M(r)$ the total (disk, bulge and halo) mass that is inside the $r$ radius, all frequencies for particles around $r$ are to increase proportionally to $\sqrt{M(r)}$. We will study the evolution of $M_{\text {inner }}=M(2 \mathrm{kpc})$, for $2 \mathrm{kpc}$ is generally about the mean radius of the bar components (whereas the maximum of the potential harmonics are around the bar extremity).

We obtain the expected increase in $M_{\text {inner }}$ (see Fig. 8). The aspect of this growth, regular or not, proves that there is a link between it and bars succession. For model A, each bar episode

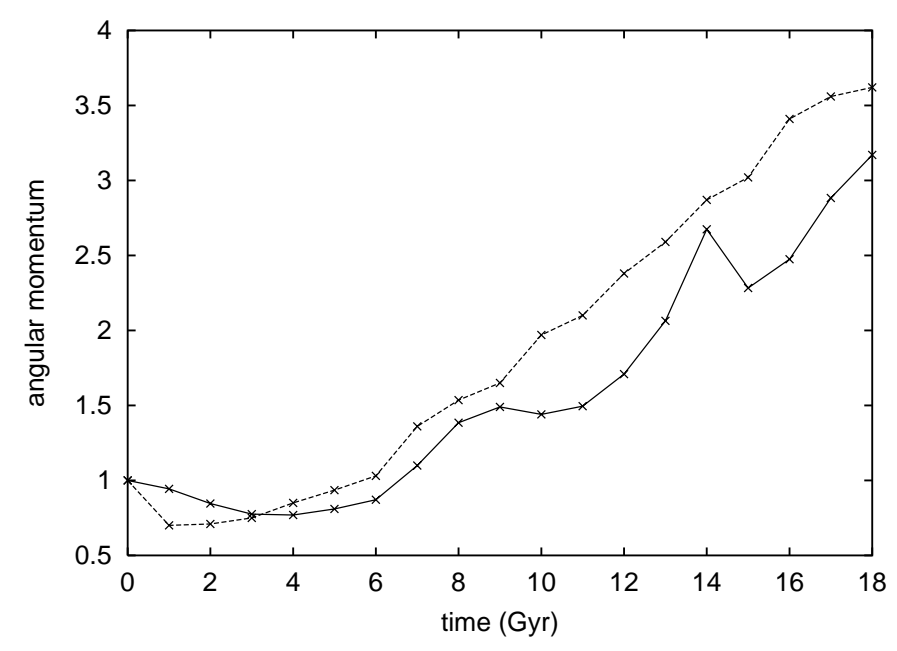

Fig. 9. Inner $(r<2 \mathrm{kpc})$ stellar and gaseous particles angular momentum per unit mass, normalized to its value at $t=0$, for model $\mathrm{A}$ (full line) and model B (dashed line).

makes $\dot{M}_{\text {inner }}$ raise: between two bars, much accreted mass comes inside the corotation, then the next bar exerts tangential forces that make this mass fall into the central condensation. For model B, $\dot{M}_{\text {inner }}$ is rather constant: accreted gas crosses the corotation in a permanent way, and supplies the central condensation during the whole galactic evolution. Let us note that $\dot{M}_{\text {inner }}$ is considered as constant (for model B), while it is actually smaller during the 6 or 7 first Gyrs. Yet, this is explained by the absence of particles accreted before $t=0$ : most of the actually accreted particles only come into the inner disk after 6-7 Gyr, and only few of them go into the central condensation earlier. So, this change in $\dot{M}_{\text {inner }}$ is linked to the beginning of accretion at $t=0$, it has no physical meaning, and what is significant is that $\dot{M}_{\text {inner }}$ is constant for $t>7 \mathrm{Gyr}$.

Accretion is responsible for an increase in $M_{\text {inner }}$. But this increase is not sufficient to explain the whole bar acceleration. For instance, the growth rates of $\sqrt{M_{\text {inner }}}$ and of $\Omega_{\mathrm{b}}$ for model A, between several instants, are given in Table 4 . The increase rate of $\sqrt{M_{\text {inner }}}$ is not sufficient, and is only partly responsible for the increase in $\Omega_{\mathrm{b}}$ : the growth rate $\Omega_{\mathrm{b}}$ is twice bigger. The bar pattern speed is also influenced by another phenomenon.

Accretion and angular momentum supply. In non accreting galactic disks, non axisymmetric fields provoke angular momentum transfers from bar to gas outside the corotation, and from gas inside the corotation to bar. In model A, the gas density is high outside the corotation when there is a strong bar, then the bar is expected to give some momentum to outer particles. When the bar strength is lower, much gas arrives in the inner disk, and may bring there its angular momentum. This is observed in Fig. 9. For model B, the accreted gas density evolves more regularly, and the angular momentum per unit mass raises steadily.

Gas accretion has therefore the main implication to raise the inner angular momentum per unit mass. If $\Omega$ is higher for a particle, its orbit's precession rate will also be faster, and it is likely that the bar instability it will participate in, will have 

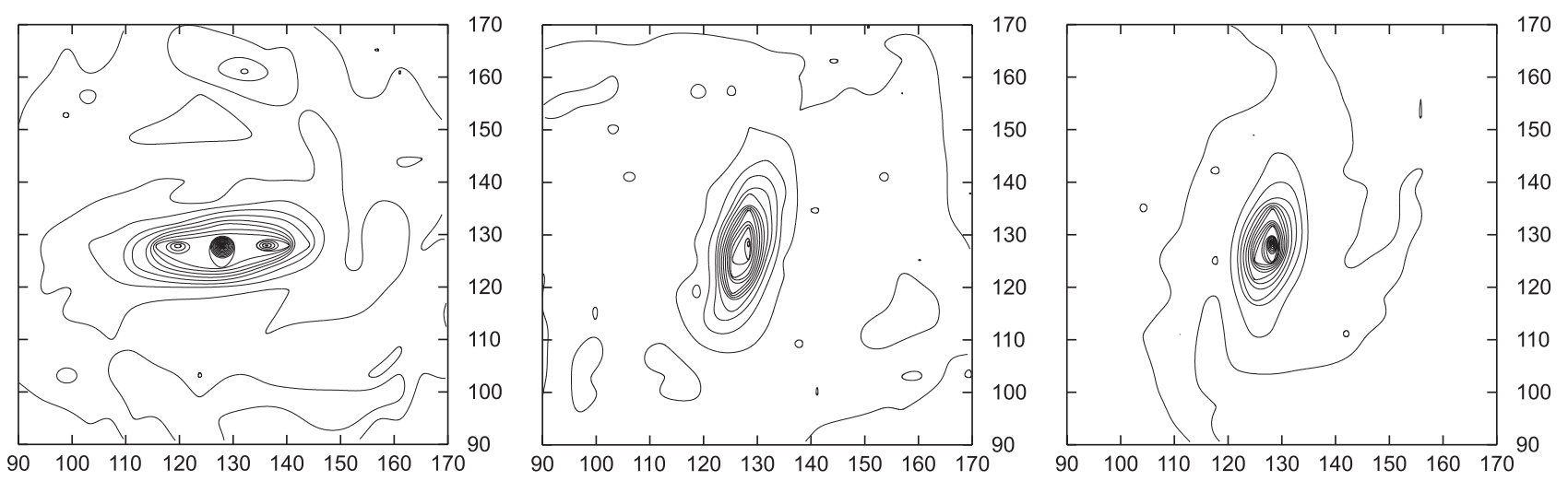

Fig. 10. Inner disk gaseous and stellar density for model A, seen at 3,10 and 15 Gyr. The unit of length is $300 \mathrm{pc}$.

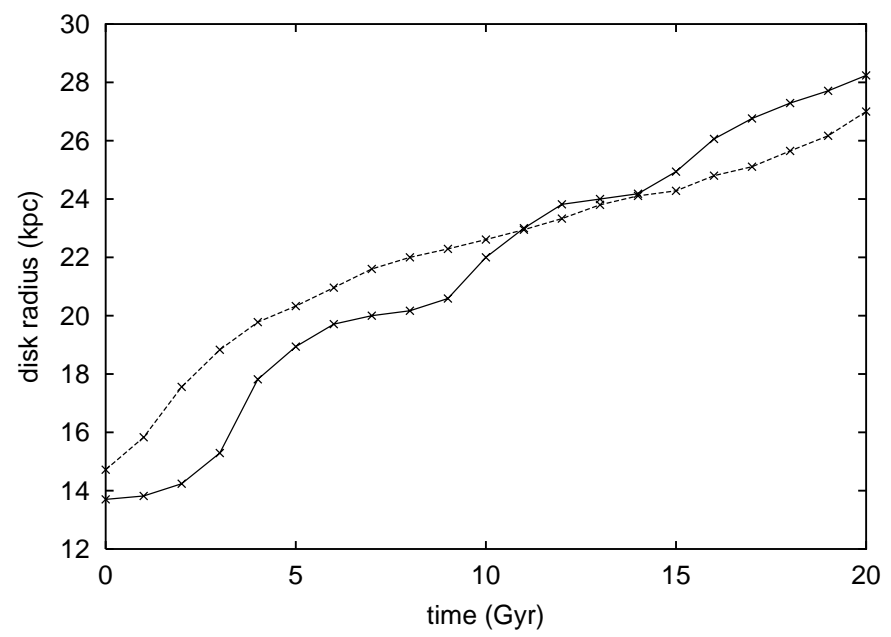

Fig. 11. Disk radius as a function of time, for models A (full line) and B (dashed line).

a higher pattern speed. The angular momentum supply makes the bar pattern speed raise.

Moreover, gaining angular momentum should make the bar become more circular, because faster particles have less eccentric orbits. This is observed in Fig. 10 for model A, and this phenomenon is similar for model B.

The main implications of gas accretion are therefore an increase of the central mass and of the angular momentum supply, and a higher bar pattern speed. Moreover, the bar becomes rounder as it gains angular momentum.

\subsubsection{Evolution of the disk radius}

The last point to discuss about models A and B is the disk radius augmentation. This is plotted in Fig. 11. Here, the disk radius is defined as the radius containing 90 percents of the disk mass.

The stronger the bar, the faster the radius grows. For both models, the OLR is outside the disk. Yet, bar torques may not explain totally the radius growth, for at least two reasons:

- even if they could enlarge outer particles orbits, they would have the same consequences without any gas accretion, which is not observed;
- when models are studied in which the OLR is inside the disk, the bar torques could only make the disk radius decrease. Yet, we still observe a radius increase.

The likely explanation is rather that the stronger the bar, the more accreted gas is accumulated in the outer disk: this gas spreads through the disk because of its dissipation; a part goes inside the corotation, and a part makes the disk expand.

\subsubsection{Bar pattern speed and corotation radius}

We have shown that gas accretion makes the bar pattern speed change. Yet, this does not imply that any change could be easily observed in real disks: the bar pattern speed cannot be directly observed, and the corotation radius is measured instead. So, we may wonder how the corotation radius evolves, and compare it to the disk radius. For instance for model B, the corotation radius and the corotation to disk radius ratio are given in Fig. 12.

The corotation radius decreases with time (see Fig. 12). This can be interpreted in terms of bar pattern speed: we have noticed that the pattern speed $\Omega_{\mathrm{b}}$ raises with time faster than the square root of the inner mass, thus faster than the particles angular velocity $\Omega(r)$. Then, the corotation radius $R_{\mathrm{cor}}$, defined by $\Omega_{\mathrm{b}}=\Omega\left(R_{\text {cor }}\right)$, is to decrease with time. Moreover, we know that the disk radius becomes larger, so that the corotation to disk radius ratio, which is the more relevant parameter, decreases in a significant way, as displayed in Fig. 12.

The bar pattern speed evolution is related to a change in the corotation radius, and makes the corotation to disk radius ratio decrease with time. Moreover, even if the bar pattern speed does not change a lot during the first Gyrs (see Fig. 7), the change in the corotation radius is larger during the first Gyrs (see Fig. 12); then the position of the corotation is significantly modified by gas accretion even in rather young disks.

\subsection{Gas accretion and spiral morphology}

We have already briefly noticed that gas accretion rejuvenates spiral patterns. In an accreting disk, the main structure of the outer disk consists in spiral arms, whereas in non accreting disks, such a structure is rather evanescent: it is replaced by circular structures in about $8 \mathrm{Gyr}$. 

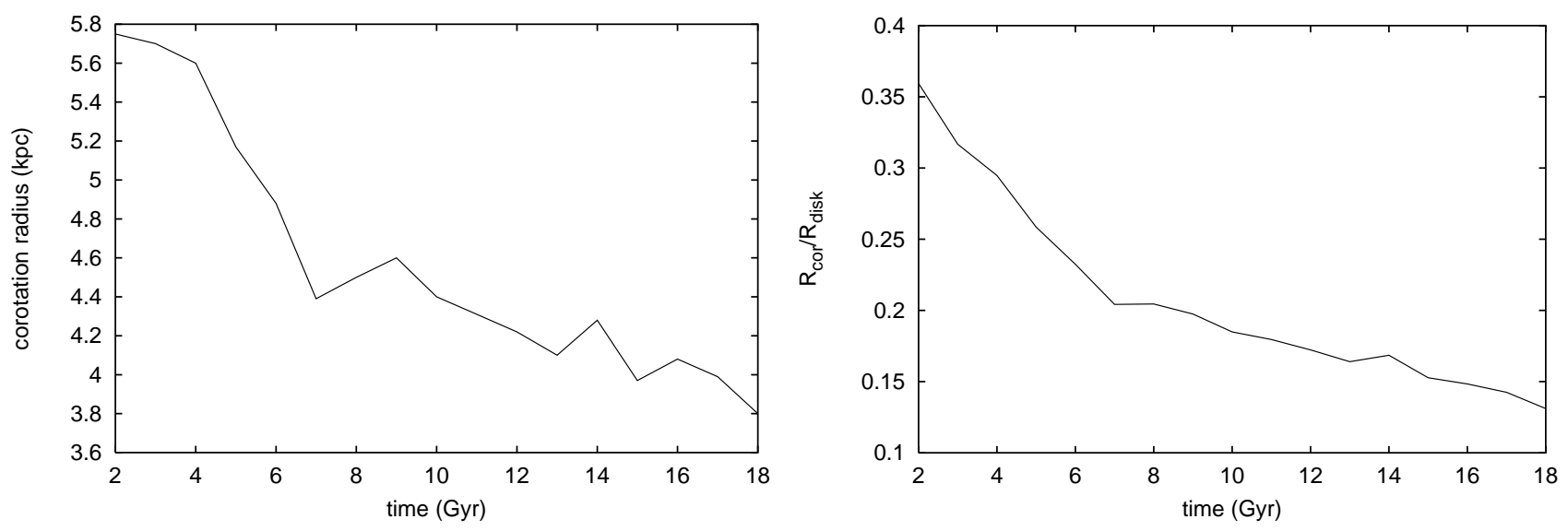

Fig. 12. Corotation radius (left) and corotation to disk radius ratio (right) for model B.

Actually, the gas mass fraction is obviously higher; so the stellar and gaseous medium are globally cooler: this prevents the stability parameter $Q$ from increasing too much, so that the spiral structure persists in the disk. When there is no accretion, the first density waves heat the medium, which does not cool back fast enough because of lack of gas.

Moreover, the accreted gas spreading prevents the disk from getting almost empty of gas in specific regions (even if does not prevent rings from appearing, as will be shown later). The initial gaseous density radial distribution is better preserved, except a change in its scale length. This lets the gaseous medium stay more favourable to spiral waves propagation.

Rejuvenating the spiral structure does not prevent resonant ring formation. Such rings may even be stronger. External rings are intensified by accreted gas accumulation in the outer disk. Inner rings, connected to ILR, do not seem to be affected by accretion. The most favored rings are those that surround the bar: they are related to the $4 / 1$ resonance. They are weaker than other rings in non accreting disks, due to gas depletion near corotation. Accretion lets them become stronger. The subsequent disks are then typically $\mathrm{RSX}, \mathrm{SX}(r)$, or even $\mathrm{RSX}(r)$ (X meaning $\mathrm{A}, \mathrm{B}$ or $\mathrm{AB}$ ) in the de Vaucouleurs classification. In this classification, the meaning of SA, SAB and SB has already been explained (see Sect. 3.1). In a SA disk, the residual bar strength, as defined in Sect. 3.2 is less than 0.2, In SAB disks, the bar strength is contained between 0.2 and 0.5 ; and in SB disks, it is more than 0.2 and can be much larger. We notice that for moderate bar strengths $(0.3-0.4)$, the disk can be either SAB or SB. A RSX-type disk shows an outer ring. The relations between bar strength and disk morphology have been studied by Block et al. (2001). A SX(r)-type disk shows a ring surrounding the bar: it is not an inner ring connected with ILR, but a larger ring. This $\mathrm{SX}(r)$-type is much favored by accretion.

Let us try to interpret the models in terms of this classification, exactly with the distinction between SB and SAB galaxies. In SB disks, spiral arms generally appear in phase with the bar and might have the same pattern speed; in the weaker SAB ones, spiral patterns are sometimes separated from the bar, and are likely to have a lower pattern speed.
Table 5. Rate of SB-type disks for different parameters.

\begin{tabular}{lcc}
\hline \hline Run & SB to [SAB+SB] ratio & SB global rate \\
\hline R1a-b-c & 20 to $30 \%$ & 15 to $20 \%$ \\
R2a-b-c & 40 to $45 \%$ & 25 to $30 \%$ \\
R3a-b-c & 75 to $85 \%$ & 50 to $55 \%$ \\
\hline
\end{tabular}

The two disks studied in Sect. 3.2 can be classified SA or $\mathrm{SAB}$, according to this definition, since Fig. 6 reveals an angular speed lower for arms than for bars. However, the disk sometimes presents an SB aspect, for both models A and B. But the frequency of strong bars (SB) does not exceed 30\%, and the rate of SB disks in the whole galactic evolution is less than $20 \%$, whereas observed galaxies are divided equally between the three categories, thus half of barred galaxies are of SB-type.

In fact, the frequency of SB disks in simulations depends on some input parameters. The halo to disk and bulge to disk ratios do not intervene there, neither does $a_{\text {bulge }}$. The important parameters are $a_{\text {disk }}$ and $a_{\text {halo. }}$ It is to be noted however that when $a_{\text {halo }}$ is varied, $M_{\text {halo }}$ is varied also in order to keep $M_{\text {halo }}^{\text {inner }}$ constant, which is the relevant mass. Moreover, increasing $a_{\text {halo }}$ is equivalent to decrease $a_{\text {disk }}$. The runs R1a to R3c are then used here, and the frequency of SB disks as a function of both scale-lengths is given in Table 5. Approximatively half of barred disks are of SB-type, and a third of disks are SA, a third $\mathrm{SAB}$, and a third $\mathrm{SB}$, which is in good agreement with observations.

Does accretion have some influence on the SB to SAB ratio (obviously, it reduces the $\mathrm{SA}$ rate, and raises the $\mathrm{SAB}$ and SB global rate, for it favours bars)? If we stop accretion at any epoch of simulation, the SB frequency decreases; furthermore, non accreting disks have lower SB frequencies than accreting ones. So, gas accretion favours a high SB to SAB ratio. Accretion brings mass in the outer disk, which makes $a_{\text {disk }}$ rise, whereas in non accreting disks, the bar makes a heavy central condensation, which on the contrary reduces the disk scalelength. Finally, gas accretion favours not only bars, but also strong SB bars. 
Table 6. Parameters of the 3-D runs with in-plane accretion. The value of $M_{\text {halo }}^{\text {inner }} / M_{\text {disk }}^{\text {initial }}$ is fixed at 0.75 . The scale-length for the disk is $5 \mathrm{kpc}$, for the bulge $1 \mathrm{kpc}$, and for the halo $20 \mathrm{kpc}$. The accretion rate makes the disk mass double in 7 Gyrs, and the initial gas fraction is $20 \%$.

\begin{tabular}{cc}
\hline \hline Run & $M_{\text {bulge }} / M_{\text {disk }}^{\text {initial }}$ \\
\hline 1 & 0.8 \\
2 & 0.6 \\
3 & 0.3 \\
\hline
\end{tabular}

\section{Three-dimensional effects of gas accretion}

We now present the results of 3-D simulations concerning bar evolution. New free parameters arise, in particular the angle between the angular momentum of the accreted gas and that of the disk. Also, the gas can be accreted at any radius. For the sake of comparison with the previous 2-D-results, let us begin by in-plane accretion.

\subsection{In-plane accretion}

In these first runs, as in the 2-D-case, the gas is assumed to be accreted in the plane, at all azimuths in the disk, with the tangential velocity corresponding to the rotation curve. This situation corresponds to a long-term, quasi-stationary accretion over a Hubble time, as if the optical disk was surrounded with slowly infalling gas, that has already settled into a larger-scale disk. Parameters of the runs are given in Table 6.

For runs 1 and 2, we observe bar destruction and reformation. These runs show respectively 2 and 3 successive bars over 15 Gyr. Run 3 presents a long-lifetime bar, that is still present in the disk after 13 Gyr. The bar accelerates such that its pattern speed doubles in about a Hubble time. The accreted gas distribution in the disk, related to bar destruction and re-formation in runs 1 and 2, is similar to that obtained in 2-D models. The vertical distribution of particles is not modified by gas accretion in these runs. Therefore, the results and interpretations reported for 2-D simulations are strengthened by these new runs.

\subsection{Out-of-plane accretion}

When gas is not accreted in the galaxy plane, we define $R_{\text {acc }}$ the ratio between the accreted gas mean radius and the disk radius (see Fig. 13). The angle between the accreted gas angular momentum and the initial disk angular momentum is $\psi_{\text {acc }}$. The accreted gas mean initial velocity has again no radial component. It seems reasonable to assume that $R_{\text {acc }}$ and $\psi_{\text {acc }}$ are nearly constant over a few Gyrs, but not over a Hubble time. Therefore, the accretion phase lasts only 5 Gyrs. It is started well after the first relaxation phase, when a first bar develops in the initial disk; a few Gyrs later, the bar weakens, and without gas accretion, the galaxy would not be strongly barred any more. We hereafter note $t=0$ the beginning of the accretion event, and not the beginning of the whole disk evolution. The accretion does not take place from all azimuths around the disk (at radii around $R_{\text {acc }}$ ), but only in a 3 -kpc-sized area, which

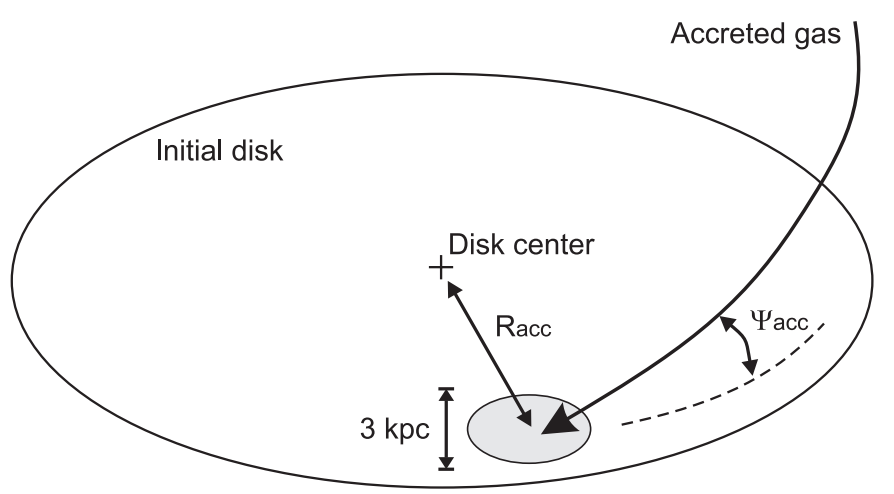

Fig. 13. Model for gas accretion and parameters $R_{\text {acc }}$ and $\psi_{\text {acc }}$.

Table 7. Parameters varied for out-of-plane accretion. $R_{\text {acc }}$ is the ratio between the accreted gas radius and the disk radius, and $\psi_{\text {acc }}$ is the angle between the angular momenta of the accreted gas and the initial disk, as described in Fig. 13. We choose $M_{\text {halo }}^{\text {inner }} / M_{\text {disk }}^{\text {initial }}=0.75$.

\begin{tabular}{cccc}
\hline \hline Run & $R_{\text {acc }}$ & $\psi_{\text {acc }}$ & $M_{\text {bulge }} / M_{\text {disk }}^{\text {initial }}$ \\
\hline REF & & no accretion & 0.8 \\
ACC8-0 & 0.8 & $0^{\circ}$ & 0.8 \\
ACC8-8 & 0.8 & $8^{\circ}$ & 0.8 \\
ACC8-20 & 0.8 & $20^{\circ}$ & 0.8 \\
ACC8-32 & 0.8 & $32^{\circ}$ & 0.8 \\
ACC8-45 & 0.8 & $45^{\circ}$ & 0.8 \\
ACC8-65 & 0.8 & $65^{\circ}$ & 0.8 \\
ACC8-87 & 0.8 & $87^{\circ}$ & 0.8 \\
ACC8-20-2 & 0.8 & $20^{\circ}$ & 0.6 \\
ACC8-20-3 & 0.8 & $20^{\circ}$ & 1.0 \\
ACC9-20 & 0.9 & $20^{\circ}$ & 0.8 \\
ACC7-20 & 0.7 & $20^{\circ}$ & 0.3 \\
ACC6-20 & 0.6 & $20^{\circ}$ & 0.3 \\
ACC3-25 & 0.3 & $20^{\circ}$ & 0.8 \\
\hline
\end{tabular}

seems more realistic for out-of-plane accretion. The mass accreted over 5 Gyrs is half of the initial disk mass. The disk evolution is followed during the accretion episode and later. Two important parameters, $\psi_{\mathrm{acc}}$ and $R_{\mathrm{acc}}$, are varied. Parameters of the run are given in Table 7.

\subsubsection{Large-radius accretion}

In runs ACC8-0 to ACC8-87, the gas is accreted in the outer disk with several different values of $R_{\text {acc }}$. The gas accretion always re-inforces the bar significantly. Warps are also formed in the outer disks, while the initial disk axis rotates.

General disk evolution. When gas is accreted with an angular momentum inclined to that of the disk, it is submitted to restoring forces from the disk, that make the orbits precess. The precession is differential, and the various gaseous orbits, at different radii will soon intersect, and dissipation will occur and make newly accreted gas align to the disk plane. Therefore all the matter will gather in a disk, which axis will slowly change 

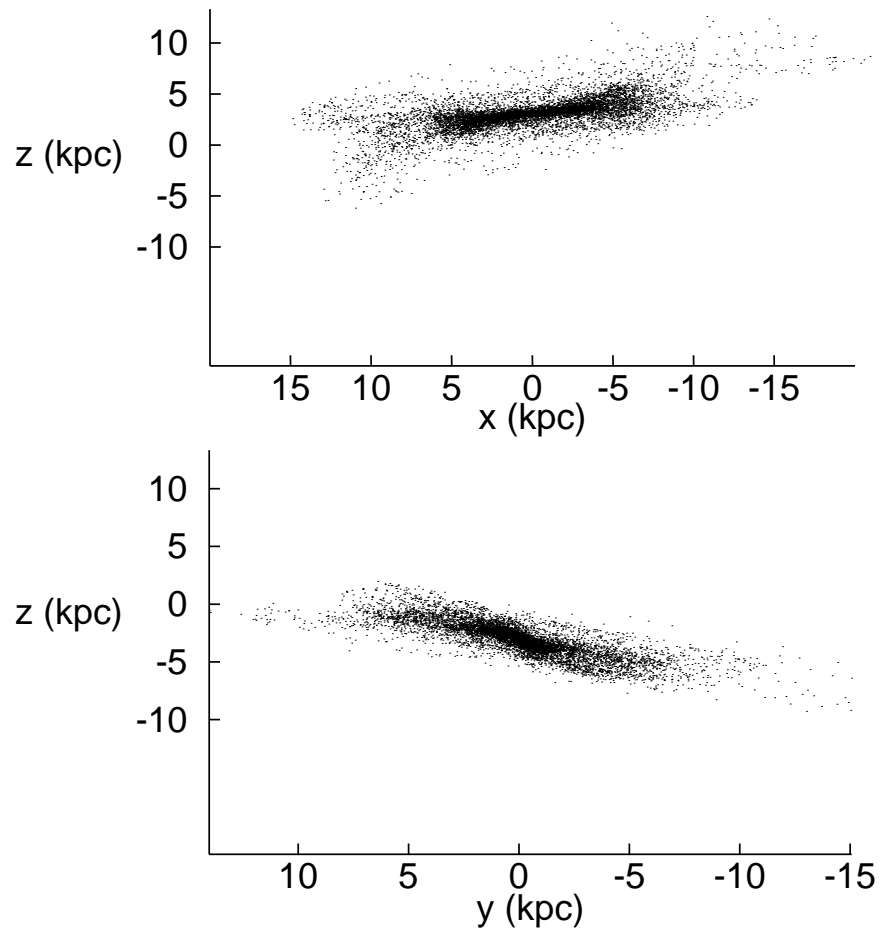

Fig. 14. Edge-on views of the gaseous component at $t=6 \mathrm{Gyr}(1 \mathrm{Gyr}$ after the end of accretion) for run ACC8-45. The view axis is perpendicular to the major axis of the bar (top), then parallel to it (bottom). For this run, the change in disk orientation is almost over at $t=6 \mathrm{Gyr}$.

orientation in a few Gyrs (see Jiang \& Binney 1999). This change in the disk orientation is observed in the present simulations (see Fig. 14). There is also a flaring in the re-oriented gaseous disk, while this flaring disappears during the change of orientation. A polar ring also appears in run ACC8-87.

Bar strength and out-of-plane accretion. For runs ACC8-8 to ACC8-87, a bar appears in the disk. It is very long around $t=$ $3-5$ Gyr, as shown in Fig. 15 for run ACC8-45 at $t=5$ Gyr. For this runs, the maximal bar strength is 0.75 , and exceeds 0.85 for runs ACC8-0, 8 and 20. In the non-accreting disk REF, the primordial bar is weakened at $t=0$ and the bar strength is never more than 0.3. Bars re-formed by out-of-plane gas accretion are much stronger than the bar re-formed in the in-plane accretion runs, where the bar strength never exceeded 0.5-0.6 in either 2-D or 3-D runs, whatever the different parameters were. The lower strength of previous bars are obvious when comparing Figs. 15 and 2.

Thus the present out-of-plane gas accretion model appears more favourable to barred waves than the previous in-plane accretion models. To interpret this, let us consider the run ACC8-0: this disk also develops a very strong bar, of maximal strength 0.9 , while the angle of accretion is zero. In fact, the common new feature introduced in the 3-D runs is to limit the accretion to a 3 -kpc-sized area of the disk. This increases the non-axisymmetric component of the gravitational potential and favors a stronger bar. Moreover, when $\psi_{\text {acc }}>0$, the accretion paths intersect the disk plane around two

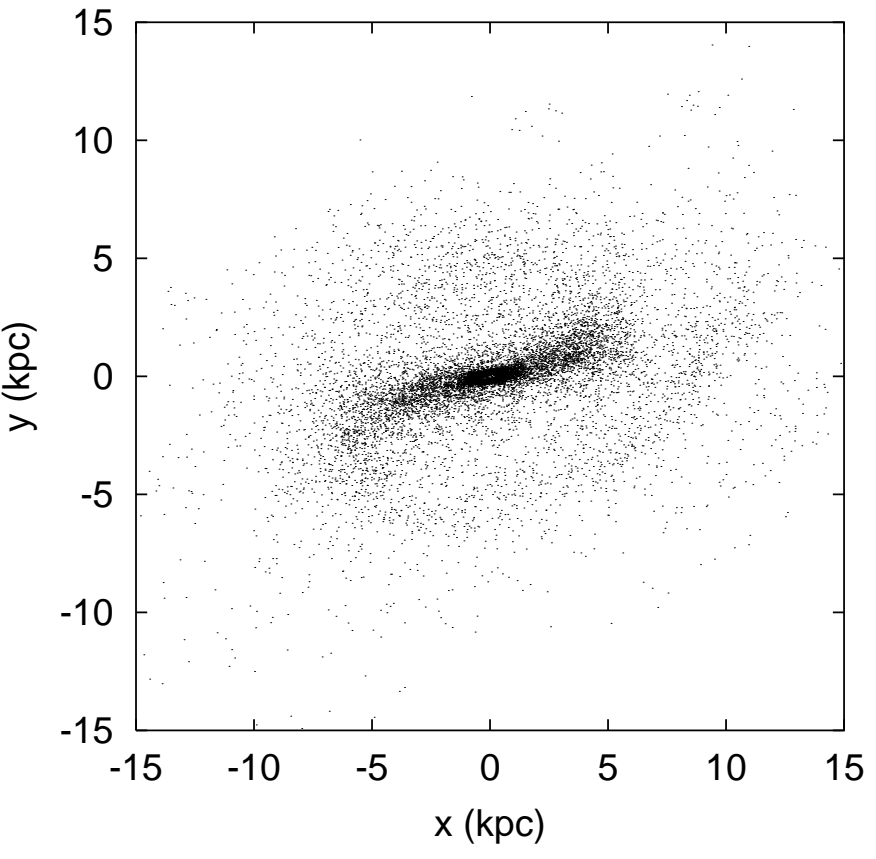

Fig. 15. Stellar and gaseous particles viewed perpendicular to the reoriented disk at $t=5 \mathrm{Gyr}$, i.e. the end of the accretion event, for run ACC8-45. This disk shows two spiral arms in phase with a very strong bar.

points: this strengthens $m=2$ harmonic of the potential, which may explain the enhancement of two spiral arms in the disks.

In summary two phenomena may explain why gas accretion favor bars in disk galaxies:

- the supply of mass to the disk, which reduces the relative mass of the central stabilizing condensation;

- the asymmetric distribution of the accreted gas.

It is difficult to disentangle the dependence of each of them on the accretion parameter $\psi_{\text {acc }}$, since both phenomena occur simultaneously. Yet, the main trends are:

- with larger $\psi_{\text {acc }}$, the gas accretion onto the disk is longer and slower, which does not favor bar formation;

- with larger $\psi_{\text {acc }}$, the distribution of the accreted gas is more asymmetric, and the $m=2$ harmonic of the resulting potential is higher.

The two mechanisms to form, re-form, or strengthen a bar, have thus opposite dependence on $\psi_{\text {acc }}$. The evolution of the actual bar strength on $\psi_{\text {acc }}$ will allow us to determine which is prevailing. The bar maximal strength as a function of $\psi_{\text {acc }}$ for runs ACC8-0 to 45 is plotted in Fig. 16.

We find that the maximal bar strength decreases when $\psi_{\text {acc }}$ increases. It appears that the influence of a strong and rapid mass supply dominates the process of bar re-formation.

Lifetime of the bar and gas accretion. Let us now examine the dependence of the bar lifetime on $\psi_{\text {acc }}$. Considering that the bar is destroyed when its strength drops below 0.2 , the lifetime of the bar in each run is shown in Fig. 17. The first point is that all these bars are rather short-lived features. This is not 


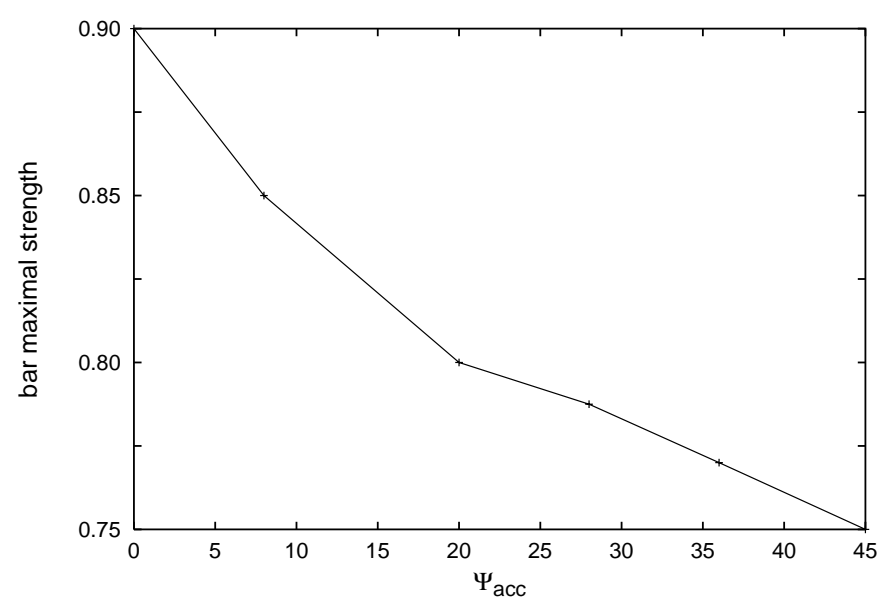

Fig. 16. Maximal bar strength over the whole bar evolution, as a function of $\psi_{\text {acc }}$, with $R_{\text {acc }}=0.8$.

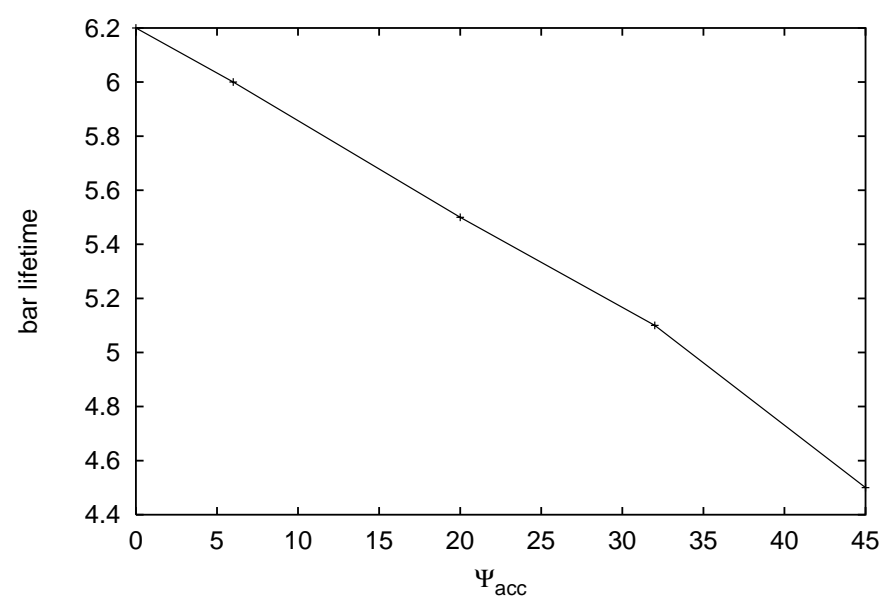

Fig. 17. Lifetime of the bar as a function of the accretion parameter $\psi_{\text {acc }}$. The bar episode ends when the bar strength is less than 0.2 . The mean accretion radius is $R_{\text {acc }}=0.8$

surprising, for at least two reasons: first the gas accretion is supposed to be limited in time, and occur during only a few Gyrs; second the formed bars are very strong, so the induced mass transfers destroy them in only a few Gyrs. The second point is the dependence of the bar lifetime with the accretion angle. The bar lifetime is longer for smaller $\psi_{\mathrm{acc}}$, and therefore the strongest bars have here the longest lifetimes. It is wellknown that in non-accreting disks, the strongest bars are the most evanescent; thus the bar lifetime is strongly influenced by gas accretion. A low $\psi_{\text {acc }}$ favors the mass supply to bars, which lengthens their lifetime. Accretion not only creates a bar, but also maintains it.

Evolution of the bar pattern speed. The evolution of the bar pattern speed with time is shown for different runs in Fig. 18. Two steps are clearly seen: when the bar is rather young (i.e. before 2-3 Gyrs), the pattern speed rises. Then, after a peaked maximum, it decreases while the bar is destroyed. Without accretion, the bar rotation would always slow down (Combes \& Sanders 1981); the acceleration observed here at the beginning is related to gas accretion. This succession of

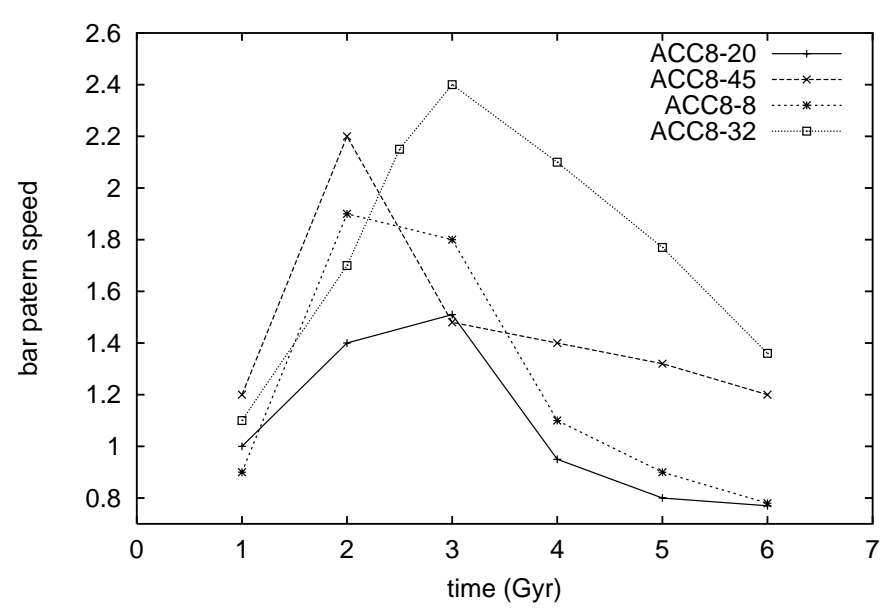

Fig. 18. Bar pattern speed as a function of time for several runs.

acceleration and deceleration of the bar rotation is similar to that observed for 2-D models with bar destruction and reformation (see Fig. 7).

In summary, out-of-plane gas accretion, like in-plane accretion, forces the bar pattern speed to increase, while bars naturally tend to slow down.

\subsubsection{Small-radius accretion and dependence on accretion radius}

When gas is accreted from outside the plane, the choice of accretion radius $R_{\text {acc }}$ is free. In the simulation ACC 3-25, the accretion radius is chosen very small. The disk radius decreases in a few Gyrs, and no bar is formed in the disk. However such a situation is rather unusual, since gas arriving at larger radii will be accelerated in the infall, and the accretion will take a long time. A rapid deceleration requires violent conditions and strong dissipation, that occur in galaxy collisions and mergers. A very small radius of accretion with high mass accretion rate is not likely to resemble a quasi-steady accretion.

Let us now study the influence of the accretion radius when $R_{\text {acc }}$ is of the same order as $r_{\text {disk }}$ : the runs involved are ACC6-20, 7-20, 8-20 and 9-20. There is no significant dependence of the bar evolution on $R_{\text {acc }}$. In fact, in the above runs, a weakened lens-shaped bar is present in the disk when we start accreting gas: its corotation radius is $7 \mathrm{kpc}$. Then, for runs ACC6-20, 7-20, 8-20 and 9-20, the gas is accreted outside the corotation (the accretion radius is more than $7.5 \mathrm{kpc}$ in those runs). As we know from 2-D studies that the important factor is the relative position of the accreted gas with respect to corotation radius, we can understand that the accretion radius does not influence the bar evolution, provided that this accretion radius is larger than the corotation one.

\subsection{General situations}

There are many free parameters concerning the way gas is accreted on a galactic disk. We have not studied every possible situation, yet we have presented several models that enables us to describe more general situations. A real disk is thought to 
accrete matter both from its plane and outside it, with parameters varying with time. Thus, a real accretion is likely to be a succession of elementary events, each of them being represented by one of our models. To illustrate this, we now present the evolution of a disk that accretes gas in its plane over a Hubble time, and from out of its plane during 5 Gyrs at the middle of its life.

In this run, the initial bulge to disk mass ratio is 0.7 , and the halo to disk is 0.75 . In-plane accretion is implemented as before with a rate that makes the initial disk mass double in 10 Gyrs. From $t=6 \mathrm{Gyr}$ to $t=11 \mathrm{Gyr}$, out-of-plane accretion takes place with $R_{\text {acc }}=0.8, \Psi_{\text {acc }}=25^{\circ}$, and a mass flow corresponding to the initial disk mass in 8 Gyrs.

Before $t=6 \mathrm{Gyr}$, the bar evolution is rather similar to the $2-\mathrm{D}$ run A. Here, the initial bar is very weakened at $t=5 \mathrm{Gyr}$, while its strength had reached 0.68 . Then, a new bar appears in the disk around $t=6 \mathrm{Gyr}$ (see Fig. 19). This bar is initially rather weak, then out-of-plane accretion strengthens it, as it has already been shown. The maximal bar strength is 0.92 . In the out-of-plane runs with a maximal bar strength larger than 0.8 , the bar strength drops below 0.4 in about 2 Gyrs. Yet, the very strong bar formed by out-of-plane accretion is here maintained by in-plane accretion. For instance, at $t=10.5 \mathrm{Gyr}$, the bar has the same aspect as at $t=8 \mathrm{Gyr}$, as shown in Fig. 19, and is bar strength still is 0.85 . Later, a bar whose strength is larger than 0.34 remains until the end of the simulation, at $t=15 \mathrm{Gyr}$.

Concerning the evolution of the bar pattern speed (normalized to 1 at $t=0$ ), the second bar formed around $t=6 \mathrm{Gyr}$ is faster than the initial one (1.36), and the pattern speed is triggered by the out-of-plane event around $t=8 \mathrm{Gyr}$ (2.35). After being very strong, the bar first slows down (1.97), but is then reaccelerated by the maintenance effect, while it becomes shorter, as in the 2-D run B: the bar pattern speed at $t=15 \mathrm{Gyr}$ is finally 2.31. Thus, the bar pattern speed doubles or even more over a Hubble time, as was the case for runs A and B (Sect. 3.2).

\section{Discussion of numerical models}

General discussions are given in Sects. 5.1 and 5.2. A discussion of the ISM scheme is given in Sect. 5.3.

\subsection{2-D models}

It is normal to expect that gas accreting galaxies maintain a relatively high gas fraction in their disk. However, even if the disk mass doubles in a few Gyrs, the gas to total (stars + gas) mass ratio is generally not more than 0.5 : the star formation and gas consumption rate is of the same order as the accretion rate. The long-term evolution of a gas accreting disk depends not only on the dynamics of this gas, but also on the evolution of stars formed in accreted matter, particularly for the consequences on stellar bars. Mass exchanges between stellar and gaseous phases have to be treated realistically. Our simulations thus include detailed star formation and stellar mass-loss schemes. In particular, the stellar mass-loss is not approximated as instantaneous: this is rather important, since mass re-injection over several Gyrs would change radial flows of matter, which then influence the bar properties (see Jungwiert et al. 2001).
However, there are still some shortcomings in the present model, since gas is assumed isothermal and the dependence of the yield on metallicity - that evolves with time, and also with accretion - is not included; it is hoped that these are only minor effects, that will not significantly affect the mass accretion rate.

The dissipation time-scale $k$ for the gas dynamics cannot be exactly justified, even if a realistic value is of a few Gyrs. Yet, this adjustable value has no major influence on the morphology of disks. When this time-scale $k$ is varied between 5 and 10 Gyrs, the long-term evolution of the disk remains unchanged: the gas reaches an equilibrium between dissipation and heating by density waves which is not significantly influenced by the value of $k$.

\subsection{3-D models}

Our three-dimensional simulations include all the major phenomena that influence the properties of bars: gas dynamics, star formation, and stellar mass-loss and feedback. However, the resolution of our 3-D models is lower than in 2-D: the cell dimension is $450 \mathrm{pc}$ (instead of $100 \mathrm{pc}$ ). The dark matter is represented by a rigid potential, and there are still many physical processes that are simplified or ignored (the multiphase nature of the gas, for instance). May be the most relevant uncertain part is the rate of star formation, that might not be a Schmidt law.

\subsection{Gas dynamics}

Different schemes were used to treat the gas dynamics in our simulations. They have given comparable results. They do not include a precise description of the real ISM. A more precise study may treat gathering and fragmentizing of gas clouds, and energy feedback from star formation. However, a simplified description of the ISM seems to be suitable for a first approach of large-scale accretion. Actually, what may be more questionable is the dissipative scheme, for it does not conserve exactly angular momentum. Sticky-particles codes are generally thought to be rather convenient; yet, we study gas-rich galaxies, which makes such a shortcoming be suspect. We have shown that angular momentum transfers and related mass exchanges are here fundamental processes, so we have to verify that the local nonconservation of the angular momentum does not invalidate our results. This is done in Appendix A.

\section{Conclusion}

The numerical simulations presented in this work reveal how different can be the evolution of spiral galaxies with and without important gas accretion. While an isolated galaxy may spontaneously form a bar, consume its gas in stars, and when the stellar component is hot enough, see its bar weaken to a lens, and evolve definitely to an early-type system, a galaxy with significant gas accretion, can reverse this evolution, and experience several new bar episodes. The detailed processes are controlled by the strength of the bar and its gravity torques. The accreted gas may stay for a while in the outer parts of the disk, prevented to flow in by a strong bar; as soon as the bar 

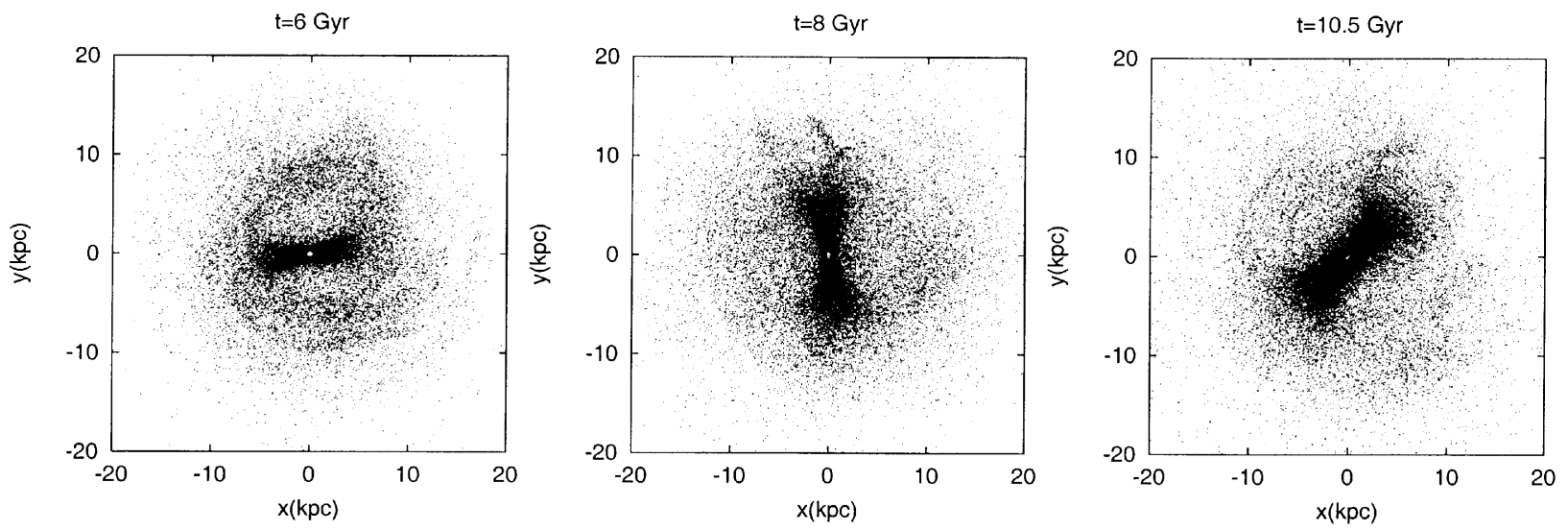

Fig. 19. Stellar and gaseous particles viewed perpendicular to the disk plane at $t=6,8$, and $10.5 \mathrm{Gyr}$, for the in- and out-of-plane accretion run.

weakens, the gas will progressively populate the disk, and form new stars there. This increases the self-gravity of the disk with respect to the stabilizing effect of the central bulge, favoring a new bar instability. Under the influence of this new bar, the material inside its corotation is driven inwards, and through vertical resonance, thickens to enrich the bulge again. This regulates the bar strength.

In the course of the simulations, three to four bar episodes have been followed. An interesting discovery is that the pattern speed of the bar is increasing form one bar to the next. This can be considered as a rejuvenating process. Indeed, the normal evolution of a bar is to slow down, while trapping more and more material, and lengthening, so that its corotation radius increases (a bar cannot extend beyond its corotation, since periodic orbits are perpendicular here). When another bar forms, it is shorter than the previous one. Its pattern speed is higher, not only because there is then more mass contained in a given radius, due to mass accretion. The pattern speed is even higher than what would be expected from the square root of mass increase. This increase may be related to the fact that mass is more and more concentrated during this evolution, and the galaxy is shifting progressively to early-types, with massive bulges. The bar is then shorter with respect to the galaxy radius (as shown by Combes \& Elmegreen 1993). Also, gas accretion has the effect of increasing the inner angular momentum per unit mass. The bar is shorter and rounder.

In the course of evolution with gas accretion, the radius of the galaxy expands, a phenomenon also controlled by the bar strength, which gravity torques can maintain or expel the gas outwards. One of the most important consequence of gas accretion, is to maintain an almost permanent (though recurrent) spiral structure through the disk, and also a significant star formation rate along a Hubble time. This solves the problem of explaining the almost constant star formation rate of spiral galaxies along their history (e.g. Kennicutt 1983), and also the presence until today of conspicuous spiral structure in most spiral disks.

The possibility to accrete gas from a direction highly inclined with respect to the galaxy plane introduces new processes that can be also favorable to bar reformation. The gas may not have to wait for a weakening of the previous bar to flow in, and the distribution is then asymmetric, with an $m=2$ extra contribution, which makes bars become much stronger.

One of the consequences of the evolution described in this work is that the bar pattern speed can no longer give the evolution state or "age" of a barred galaxy. Significant gas accretion can reverse the evolution and increase the pattern speed. Also, the argument that dynamical friction would have slowed down the bars along their evolution would have to be revised (e.g. Debattista \& Sellwood 1998). Another consequence is that the morphology of a galaxy, according to its environment, and the availability to accrete cold gas, may oscillate from late to early-types three or four times, before inexorably shift to an early-type galaxy.

The maintenance of conspicuous spiral structure in most disk galaxies may mean that galaxies have sufficient gas to accrete in their outer parts, to constantly renew disk instability, even in the presence of a heating stellar disk and increasing bulge mass. The relative absence of bars in galaxies at high redshift (e.g. van den Bergh et al. 1996) could be interpreted as a very short life-time of the bar episode, in the presence of dominating gas fraction in very young galaxies. Indeed, the fraction of barred galaxies at a given epoch is interpreted, in this recurrent bar scenario, as the relative time spent by a galaxy in the bar episode relative to the un-barred one.

The present work has only considered general properties of gas accreting spiral galaxies, but many parameters (mass distribution, morphological types, geometry of the accretion, etc.) have to be explored before all consequences of accretion on galaxies can be drawn, which will be done in future work.

\section{Appendix A: Numerical schemes for gas dynamics and non conservation of integrals of motion}

\section{A.1. Variations of angular momentum and energy}

We have shown that redistribution of angular momentum inside the disk by spirals and bars waves was partly responsible for an increase in the bar pattern speed, which is an important consequence of gas accretion. However, the numerical codes we have used do not conserve angular momentum. Both ISM models, viscosity scheme or sticky-particles code, dissipate the angular momentum of gas clouds, at least with the chosen 
Table A.1. Relative variations of angular momentum and energy of gas particles over 5 Gyrs, for the control run without accretion, star formation, and stellar mass-loss. The initial values are normalized to 1 .

\begin{tabular}{rc}
\hline \hline Integral of motion & Value after 5 Gyrs \\
\hline 2-D viscosity scheme & \\
Angular momentum & 0.97 \\
Kinetic energy & 0.60 \\
3-D sticky-particles scheme & \\
Angular momentum & 0.98 \\
Kinetic energy & 0.53 \\
\hline
\end{tabular}

parameters. This could be a real process if a spin was stored in gas particles, but it is not the case in our simulations. Thus, we discuss in this appendix the non-conservations of angular momentum by gas dynamics schemes, in relation with our results, and more generally in all $\mathrm{N}$-body simulations. Another basic integral, the energy, is not conserved in the ISM: here it corresponds to a physical process, for gas clouds radiate away a significant energy.

In a sticky-particles code, when two gas particles collide, their local angular momentum is generally not conserved. According to Eqs. (18) to (24), the angular momentum of the particles in the referential of their mass center before the collision is:

$\boldsymbol{L}=\left(\boldsymbol{r}_{2}-\boldsymbol{r}_{1}\right) \times \boldsymbol{v}_{\mathrm{t}} \frac{m_{2} m_{1}}{M}$.

After the collision, only velocities have changed, and the angular momentum is:

$\boldsymbol{L}^{\prime}=\left(\boldsymbol{r}_{2}-\boldsymbol{r}_{1}\right) \times \boldsymbol{v}^{\prime}{ }_{\mathrm{t}} \frac{m_{2} m_{1}}{M}$

$\boldsymbol{L}^{\prime}=\beta_{\mathrm{t}} \boldsymbol{L}$.

Thus, the sticky-particles code conserves the angular momentum only when $\beta_{\mathrm{t}}=1$. It does not when $\beta_{\mathrm{t}}=0.65$. However, during a collision, the angular momentum around the mass center is changed, but the momentum of the mass center around the galactic center remains unchanged. Actually, the second one is much larger: its mean value, for each pair of gas particles susceptible to collide, is almost $10^{3}$ larger than the mean value of the first one (this measure is for run $0 \mathrm{~F} 1$ ). Thus, only a small fraction of the angular momentum can be reduced. We are now to show quantitatively that the variations of angular momentum induced by both dissipative schemes are not as important as the effects of gas accretion that we have shown.

Accretion is responsible for changes in angular momentum and energy, thus, in order to quantify the non conservation of integral of motions due to our code, we compute the variation of energy and angular momentum in an isolated disk (run $0 \mathrm{~F} 1$ in Sect. 3). Star formation and stellar mass-loss modify the gas content and the importance of gas dynamics, thus we also suppress them. In Table A.1 we give the relative variation of angular momentum and energy in the gaseous disk over 5 Gyrs. The quantities taken away by particles going out of the grid have been accounted for, thus the gas dissipation is the only cause for the observed variations. The ISM undergoes an important loss of energy, that is physically justified, and a small

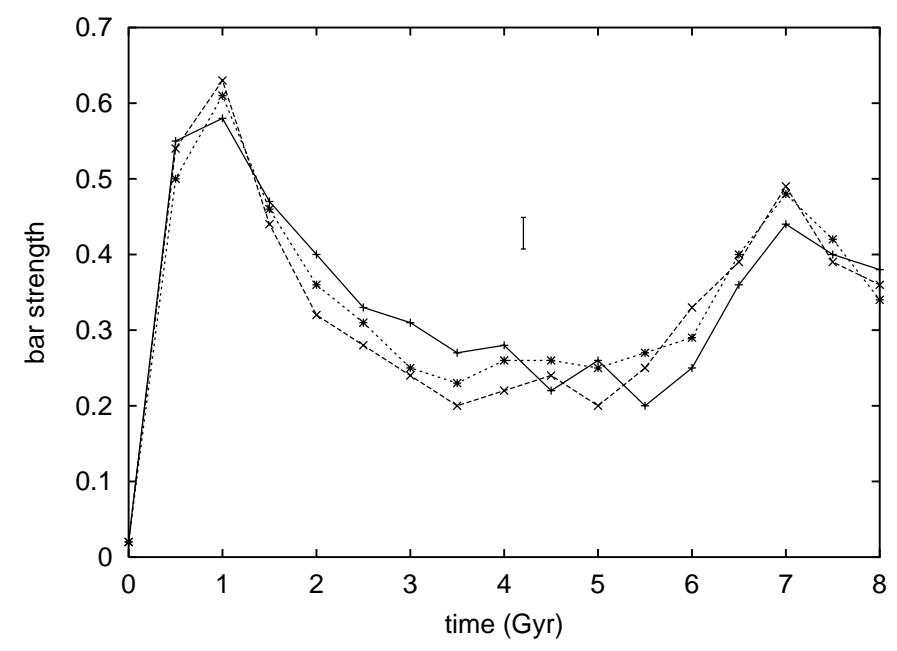

Fig. A.1. Bar strength in a 2-D simulation. Solid line: angular momentum is conserved (sticky-particles $\beta_{\mathrm{t}}=1$ ), dashed line: angular momentum is not conserved (viscosity scheme), dotted line: angular momentum is conserved (modified viscosity scheme). We show the scatter (error bar) measured on 5 simulations starting with 5 different, randomly chosen, initial position of the particles, all the physical parameters remaining unchanged.

loss of angular momentum: as explained qualitatively before, collisions in the 3-D code, and viscosity in the 2-D one, dissipate only a small fraction of the angular momentum, that cannot be compared with the large supply of angular momentum by accretion.

\section{A.2. Influence of the angular momentum conservation on previous results}

We have explained that numerical schemes for gas dynamics do not conserve exactly the angular momentum. We wish to show that this is not a critical point, and does not weaken our conclusions about gas accretion.

The dissipative schemes we have used suppress local vortices, and tend to reduce the angular momentum of two colliding particles. This may modify the dynamics of the galactic center. Thus, we modify the value of $\beta_{\mathrm{t}}$ to 1 , then the angular momentum is conserved. We test simulations with this parameter against previous results established with a sticky-particles code, i.e. 3-D results. For 2-D results, we compare the viscosity scheme to a modified version in which only the radial velocities are reduced, still in order to conserve angular momentum: in this modified version, a particle at a position $\boldsymbol{r}$ from the disk center, with a velocity $\boldsymbol{v}$, in a cell in which the mean velocity is $<\boldsymbol{v}>$, will undergo a force that reads:

$\boldsymbol{f}=-k((\boldsymbol{v}-<\boldsymbol{v}>) \cdot \boldsymbol{r}) \frac{\boldsymbol{r}}{r^{2}}$

We also compare the 2-D viscosity scheme with a 2-D stickyparticles code, using $\beta_{\mathrm{t}}=1$.

In Fig. A.1, we compare the evolution of the bar strength in a 2-D disk for the viscosity scheme, its modified version that conserves angular momentum, and the sticky-particles code with $\beta_{\mathrm{t}}=1$. Figure A. 2 shows the evolution of the bar pattern 


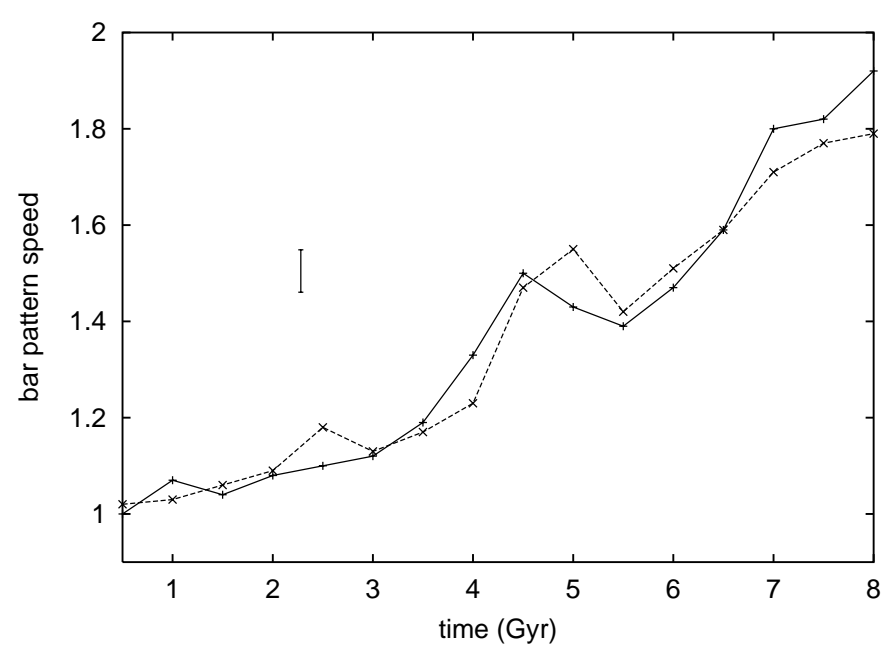

Fig. A.2. Bar pattern speed in a 3-D simulation. Solid line: angular momentum is conserved $\left(\beta_{\mathrm{t}}=1\right)$, dashed line: angular momentum is not conserved $\left(\beta_{\mathrm{t}}=0.65\right)$. The error bar has the same meaning as in Fig. A.1.

speed in a 3-D run, for the sticky-particles code with $\beta_{\mathrm{t}}=0.65$ and 1 . The results are quite similar for the different codes, and the differences between each of them is not significant: they do not largely exceed the scatters obtained on 5 simulations starting with the same values for physical parameters, but with 5 different, randomly chosen, initial positions for the particles.

For both 2-D and 3-D models, we conclude that conserving the local angular momentum or not does not significantly influence the bar evolution and the spiral structure. This confirms all our previous results. More generally, this shows that the 2-D linear dissipative scheme and the sticky-particles code are rather convenient when one studies the evolution of disk galaxies. Furthermore, the dissipation in the real ISM is likely to be somewhere in between our two schemes. One scheme exactly conserves the angular momentum, the other one annihilates local vortices: real gas clouds may dissipate a fraction of angular momentum in cloud-cloud collisions, and store it in their spin. As the results are similar with or without dissipating angular momentum, it seems likely that the response of the real ISM to gas accretion on galactic disk is similar to what we have drawn out from our simulations.
Acknowledgements. We are grateful to the anonymous referee for his/her positive criticisms that helped to improve the paper. The 3-D computations in this work have been realized on the Fujitsu NEC-SX5 of the CNRS computing center, at IDRIS. The 2-D simulations have been carried out on a PC desktop computer.

\section{References}

Briggs, F. 1990, ApJ, 352, 15

Block, D. L., Puerari, I., Knapen, J. H., et al. 2001, A\&A, 375, 761

Combes, F. 2000, in Dynamics of Galaxies from the Early Universe to the Present, ed. F. Combes, G. A. Mamon, \& V. Charmandaris, ASP Conf. Ser., 197, 15

Combes, F. 1994, in Mass-transfer induced activity in galaxies, ed. I. Shlosman (Cambridge Univ. Press), 170

Combes, F., \& Elmegreen, B. G. 1993, A\&A, 271, 391

Combes, F., \& Gerin, M. 1985, A\&A, 150, 327

Combes, F., \& Sanders, R. H. 1981, A\&A, 96, 164

de Vaucouleurs, G. 1963, ApJS, 8, 31

Debattista, V. P., \& Sellwood, J. A. 1998, ApJ, 493, L5

Eskridge, P. B., Frogel, J. A., Pogge, R. W., et al. 2000, AJ, 119, 536

Friedli, D., \& Benz, W. 1993, A\&A, 268, 65

Friedli, D., \& Martinet, L. 1993, A\&A, 277, 27

Gerin, M., Combes, F., \& Athanassoula, E. 1990, A\&A, 230, 37

Hasan, H., \& Norman, C. A. 1990, ApJ, 361, 69

Hasan, H., Pfenniger, D., \& Norman, C. 1993, ApJ, 409, 91

Hut, P., Makino, J., \& McMillan, S. 1995, ApJ, 443, 93

James, R. A. 1977, J. Comput. Phys., 25, 71

Jiang, I.-G., \& Binney, J. 1999, MNRAS, 303, L7

Jungwiert, B., Combes, F., \& Palouš, J. 2001, A\&A, 376, 85

Jungwiert, B., \& Palouš, J. 1996, A\&A, 311, 397

Katz, N., Weinberg, D. H., Hernquist, L., \& Miralda-Escude, J. 1996, ApJ, 457, L57

Kennicutt, R. C. Jr. 1998, ApJ, 498, 541

Kennicutt, R. C. Jr. 1998, ARA\&A, 36

Kennicutt, R. C. Jr. 1983, ApJ, 272, 54

Miwa, T., \& Noguchi, M. 1998, ApJ, 499, 149

Pfenniger, D., \& Friedli, D. 1991, A\&A, 252, 75

Pfenniger, D., \& Norman, C. 1990, ApJ, 363, 391

Sancisi, R. 1983, in Internal kinematics and dynamics of galaxies (Dordrecht, D. Reidel), Proc. Symp. IAU, 100, 55

Schwarz, M. P. 1981, ApJ, 247, 77

Toomre, A. 1964, ApJ, 139, 1217

Toomre, A. 1963, ApJ, 138, 385

van den Bergh, S., Abraham, R. G., Ellis, R. S., et al. 1996, AJ, 112, 359 